\title{
Time of flight mass spectra of ions in plasmas produced by hypervelocity impacts of organic and mineralogical microparticles on a cosmic dust analyser
}

\author{
B. J. Goldsworthy ${ }^{1}$, M. J. Burchell ${ }^{1}$, M. J. Cole ${ }^{1}$, S. P. Armes ${ }^{2}$, M. A. Khan ${ }^{2}$, S. F. Lascelles ${ }^{2}$, S. F. Green ${ }^{3}$, \\ J. A. M. McDonnell ${ }^{3}$, R. Srama ${ }^{4}$, and S. W. Bigger ${ }^{5}$ \\ ${ }^{1}$ Centre for Astrophysics and Planetary Science, University of Kent at Canterbury, Kent CT2 7NR, UK \\ 2 School of Chemistry, Physics and Environmental Science, University of Sussex, Falmer, Brighton BN1 9QJ, UK \\ 3 Planetary and Space Science Research Institute, The Open University, Milton Keynes, MK7 6AA, UK \\ ${ }^{4}$ Max-Planck Institute für Kernphysik, Postf. 103980, 69029 Heidelberg, Germany \\ 5 School of Life Sciences and Technology, Victoria University, Melbourne City, Australia
}

Received 4 June 2002 / Accepted 16 July 2003

\begin{abstract}
The ionic plasma produced by a hypervelocity particle impact can be analysed to determine compositional information for the original particle by using a time-of-flight mass spectrometer. Such methods have been adopted on interplanetary dust detectors to perform in-situ analyses of encountered grains, for example, the Cassini Cosmic Dust Analyser (CDA). In order to more fully understand the data returned by such instruments, it is necessary to study their response to impacts in the laboratory. Accordingly, data are shown here for the mass spectra of ionic plasmas, produced through the acceleration of microparticles via a $2 \mathrm{MV}$ van de Graaff accelerator and their impact on a dimensionally correct CDA model with a rhodium target. The microparticle dusts examined have three different chemical compositions: metal (iron), organic (polypyrrole and polystyrene latex) and mineral (aluminosilicate clay). These microparticles have mean diameters in the range 0.1 to $1.6 \mu \mathrm{m}$ and their velocities range from 1-50 $\mathrm{km} \mathrm{s}^{-1}$. They thus cover a wide range of compositions, sizes and speeds expected for dust particles encountered by spacecraft in the Solar System. The advent of new low-density, microparticles with highly controllable attributes (composition, size) has enabled a number of new investigations in this area. The key is the use of a conducting polymer, either as the particle itself or as a thin overlayer on organic (or inorganic) core particles. This conductive coating permits efficient electrostatic charging and acceleration. Here, we examine how the projectile's chemical composition influences the ionic plasma produced after the hypervelocity impact. This study thus extends our understanding of impact plasma formation and detection.

The ionization yield normalized to particle mass was found to depend on impact speed to the power $(3.4 \pm 0.1)$ for iron and $(2.9 \pm 0.1)$ for polypyrrole coated polystyrene and aluminosilicate clay. The ioization signal rise time was found to fall for all projectile materials from a few microseconds at low impact speeds $\left(3 \mathrm{~km} \mathrm{~s}^{-1}\right)$ to a few tenths of a microsecond at higher speeds (approximately $16 \mathrm{~km} \mathrm{~s}^{-1}$ for aluminosilicate particles and approximately $28 \mathrm{~km} \mathrm{~s}^{-1}$ for iron and polystyrene particles). At speeds greater than these the rise time was a constant few tenths of a microsecond independent of impact speed. The mass resolution of the time of flight spectrometer was found to be non-linear at high masses above $100 \mathrm{amu}$. It was $\Delta m / \mathrm{m}=5$ for $m=$ $1 \mathrm{amu}$ and 40 for $m=200 \mathrm{amu}$. However, although at high masses most mass peaks had the resolution quoted, there were also occasional much narrower mass peaks observed, suggesting that at 250 to $280 \mathrm{amu} \Delta m / m=80$ to 100 . The lower resolutions may be due to closely spaced mass peak signals effectively merging into one observed peak due to the (greater but still finite) resolution found for the isolated mass peaks.

Complex mass spectra have been reproducibly obtained from a number of different projectiles that display many charged molecular fragments with masses up to $250 \mathrm{amu}$ and with periodicities of 12-14 amu. These new studies reveal an extremely strong dependence of the time-of-flight mass spectra on the impact speed, particularly at low velocities $\left(1-20 \mathrm{~km} \mathrm{~s}^{-1}\right)$. In some impact velocity regimes it is possible to distinguish time-of-flight spectra originating from organic microparticles from those obtained from iron microparticles. However, such discrimination was not possible at high impact speeds, nor was it possible to distinguish between the time-of-flight spectra obtained for aluminosilicate particles from those obtained for iron projectiles.
\end{abstract}

Key words. solar system: general - interplanetary medium - instrumentation: detectors

\section{Introduction}

Send offprint requests to: M. J. Burchell, e-mail: M. J.Burchell@kent.ac.uk
The in-situ analysis of cosmic particles is a growing area of scientific interest with current interplanetary missions carrying detectors equipped with composition determining 
capabilities. One such instrument is the Cassini Cosmic Dust Analyser (CDA) (Srama et al. 2003) onboard the CassiniHuygens mission bound for Saturn. In this context, particles refer to individual grains with diameters typically a few microns or smaller. Through the collection of the plasma produced during a hypervelocity impact of such particles on a target, it is possible to determine the overall flux and to discern several key parameters of the original grain, such as mass, impact speed and composition. Flux detectors flown on satellite and interplanetary missions since the 1960's have identified many distinct dust populations within the solar system including meteoritic, cometary, interstellar and highly collimated streams found at the Jupiter system (Grün et al. 1993; Grün et al. 1998). The Divine model (Divine 1993) attempted to describe the interplanetary environment based on five original populations. This is currently being extended to include particle motions and updated to include newly observed populations such as interstellar dust (Grün \& Staubach 1996; Matney \& Kessler 1996).

More recently, as well as measuring flux, CDA relies on the formation of ionic plasma by microparticles impinging at hypervelocities to perform time-of-flight mass spectrometry analysis. It provides compositional information on encountered dust grains, which can give an insight into their origin and the physicochemical processes involved in maintaining the dustflux environment. Calibration of such instruments with a wide variety of materials and at different impact speeds is essential to develop a proper understanding of the processes involved and to enable the interpretation of returned data from space.

Laboratory simulation of micrometeoroid impacts by electrostatic acceleration of microparticles to speeds of several $\mathrm{km} \mathrm{s}^{-1}$ was first reported by Friichtenich (1962). Subsequently, observations of impact ionization were reported by Friichtenicht \& Slattery (1963), and occurred when high speed particles (greater than a few $\mathrm{km} \mathrm{s}^{-1}$ ) impacted targets. The resulting intense local heating and at least partial vaporisation of both projectile and target (at the impact site) from the impact shock produces a plasma composed of components from the target and the original projectile. It should be noted however that ionization is also obtained at speeds of less than a few $\mathrm{km} \mathrm{s}^{-1}$, i.e. below the somewhat loose traditional speed limit for full onset of hypervelocity behaviour (see Burchell et al. 1998, where ionization is observed for impacts of iron microparticles on copper targets at speeds as low as $0.9 \mathrm{~km} \mathrm{~s}^{-1}$ ). This suggests that the ionization process is more complicated than this simple model. There are several models that attempt to explain this phenomenon; although as noted the processes involved are very complex. These models have been used to suggest qualitative explanations of the production of plasma in events with high impact velocities $\left(>30 \mathrm{~km} \mathrm{~s}^{-1}\right)$. However, quantitative predictions are generally difficult (for a summary see Auer 2001). By applying an electric field within the region of plasma formation the positive and negative components can be separated and analysed for compositional information (Hansen 1968). Allowing the separated ionised component to drift for some distance produces a time-of-flight spectrum, where the more massive ions have a longer flight time in the detector. Assuming that all ion species are singly ionised, this time-of-flight spectrum can be converted into an atomic (or molecular) mass spectrum.

The technique of compositional analysis through impact ionization time-of-flight mass spectrometry forms the basis of a number of interplanetary dust detectors. It has the advantage of providing in-situ compositional analysis without requiring complex, expensive sample return missions. Additionally, it provides a tool with which to investigate the origin of the particulate population based on composition that can be incorporated into flux models. The first mission to carry such an instrument was Helios in 1974 (Dietzel et al. 1973). In 1986 three spacecraft (Giotto, VeGa 1 and 2) encountered Halley's comet, each carrying similar particle analysers, PIA and PUMA 1 and 2 (see Kissel 1986 and Kissel et al. 1986a,b). These had a small impact target area but higher spectral resolution $(m / \Delta m>150)$ than CDA. The high average encounter speed (69-80 $\left.\mathrm{km} \mathrm{s}^{-1}\right)$ at Halley's comet caused the plasma in many events to be primarily elemental, with some evidence of molecular fragments. Much analysis of the mass spectra obtained from Halley particles has been performed. From an early stage it was clear that some events were dominated by the light elements carbon, hydrogen, oxygen, and nitrogen, or so-called CHON spectra, (Kissel et al. 1986a) while others contained mainly rock-forming elements. Currently a similar instrument is aboard the Stardust mission destined to visit comet Wild 2 in 2004.

The Cassini-Huygens mission (Spehalski 1996) to explore the Saturn system was launched successfully in October 1997 and is equipped with a host of instruments, including the Cassini Cosmic Dust Analyser. This provides in-situ observations of the dust environment both during the seven-year cruise phase (where possible) and of the Saturnian system (arrival 2004). The CDA instrument is a direct descendant of the highly successful dust flux instruments carried on the Galileo and Ulysses missions (Grün et al. 1992a, 1992b respectively) with the addition of a small chemical analyser target. Shown in Fig. 1, it weighs $17 \mathrm{~kg}$ and is capable of determining particle charges between $1 \times 10^{-15}$ and $5 \times 10^{-13} \mathrm{C}$ and particle masses between $10^{-18}$ and $10^{-12} \mathrm{~kg}$ for impact speeds of 1 to $100 \mathrm{~km} \mathrm{~s}^{-1}$. For a detailed description see Srama \& Grün (1996). This instrument has a lower mass resolution than the PIA, PUMA and CIDA (Stardust) detectors. However, it has a larger chemical analyser target area of $200 \mathrm{~cm}^{2}$, compared to $5 \mathrm{~cm}^{2}$ for both PIA and PUMA and $90 \mathrm{~cm}^{2}$ for CIDA (Grün et al. 2000) combined with a very large area ionization target $\left(1000 \mathrm{~cm}^{2}\right)$ for particle flux and mass measurements; this makes it well suited to the extended cruise phase of the Cassini mission where relatively low fluxes are expected, unlike the high fluxes encountered during cometary flybys.

To fully understand data returned from CDA, it is essential that laboratory experiments are performed to characterise the instrument responses and to further understand the physical processes at work. One method used to simulate ionic plasma production from a target is laser desorption (e.g. Kissel $\&$ Krueger 1987; Ahrens et al. 2003), which is useful for timeof-flight dynamic investigations. However, this technique has the disadvantage that strictly speaking only the target material is ionised, there is no projectile present. So the behaviour of 


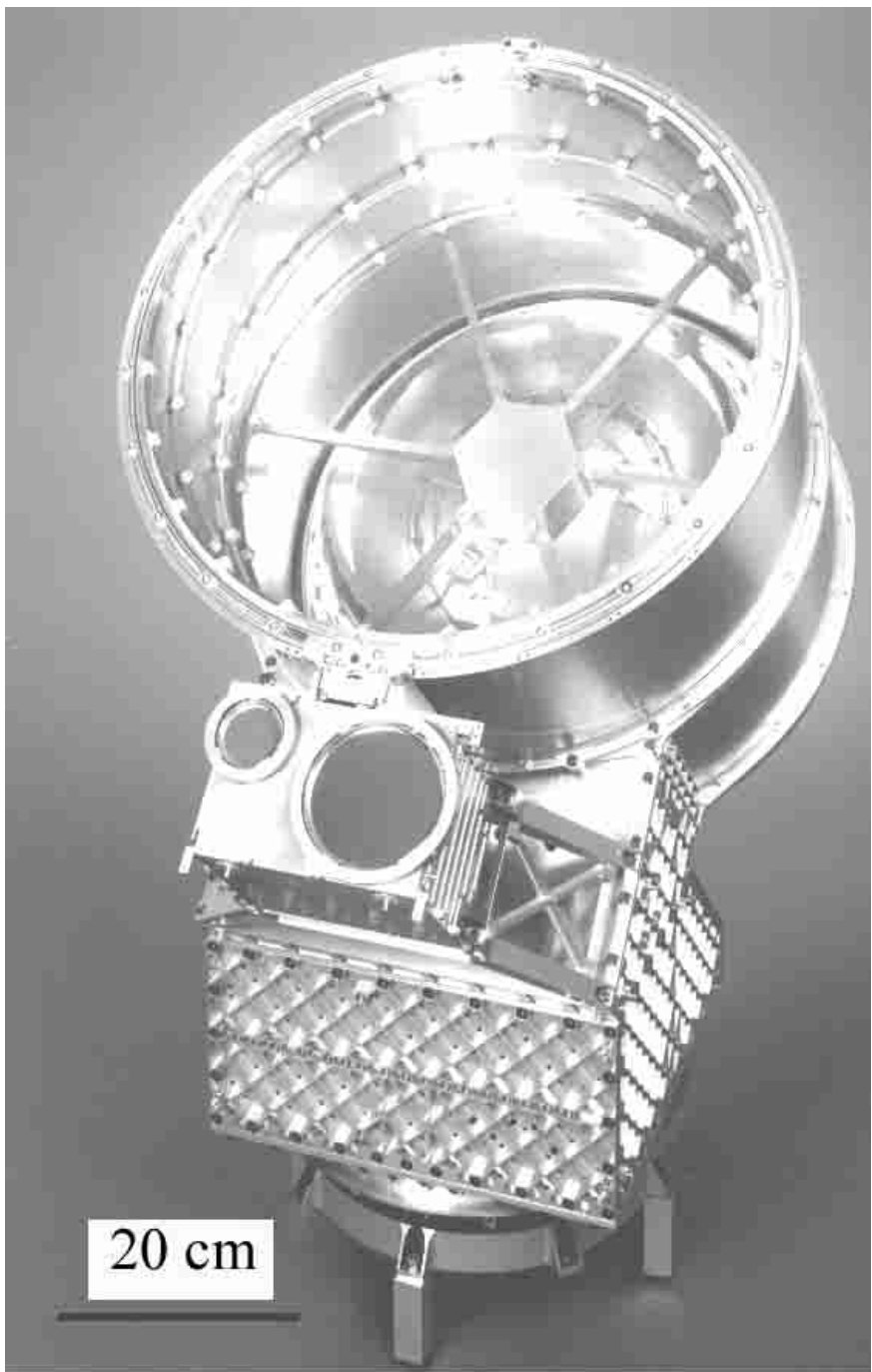

Fig. 1. Cassini Cosmic Dust Analyser flight spare, courtesy of Heidelberg Max-Planck-Institut für Kernphysik. The hollow drum like structure in the top half of the figure is the main body of the instrument. The target is at the bottom of this drum and the ion detector is mounted in the hexagonal assembly suspended in the middle of the drum. Suspended beneath the circular opening of the main detector is a rectangular box with two circles on the front face. This is a high rate dust flux monitor using pvdf impact momentum sensors.

projectile material has to be inferred. An alternative approach is to simulate dust impacts by using a van de Graaff electrostatic accelerator to accelerate dust grains to a suitable speed and impacting them upon a target. A substantial amount of previous laboratory experimentation of this type has relied on impacts of dense, metallic projectiles such as iron, gold and copper, etc., simply because they can be readily charged and are thus suitable for electrostatic acceleration. However, such projectiles are elemental in composition and are unlikely to be representative of the expected flux of interplanetary dust particles. For example, if of cometary origin, particles encountered in interplanetary space are predicted to be highly porous, have low densities and to possess organic or mineral content in many cases. In addition, previous experiments have reported ionization from impacts on a variety of target materials, for example, Burchell et al. (1999a) reports data for impacts on targets such as iron, molybdenum, indium, gold and silver as well as rhodium.

Accordingly, we have used conducting polymers of relatively low density (typically around $1100-1500 \mathrm{~kg} \mathrm{~m}^{-3}$ ) to extend the composition of microparticles available for electrostatic acceleration. These new microparticles can be synthesised reproducibly with well-defined size distributions and chemical compositions. Here we report the successful van de Graaff electrostatic acceleration of several types of conducting polymer-coated projectiles, including two different organic-based latex particles and aluminosilicate-based clay platelets, and their hypervelocity impacts onto a full-scale replica of the Cassini CDA detector are studied. The response of the target to low-density projectiles is studied and a discussion of the time-of-flight spectral features is presented, with particular emphasis being placed on their evolution with impact speed. Finally, spectral analysis provides the mass resolution of the laboratory-based instrument for ionic species with masses in excess of $100 \mathrm{amu}$.

\section{Experimental procedure}

A $2 \mathrm{MV}$ van de Graaff accelerator at the University of Kent at Canterbury was used to accelerate electrically conducting microparticles up to speeds that are representative of dusts encountered in interplanetary space $\left(1-50 \mathrm{~km} \mathrm{~s}^{-1}\right)$. The accelerator facility and dust source are detailed in Burchell et al. (1999a). The accelerated particles impacted upon a CDA representative laboratory model in an environment evacuated to a pressure lower than $10^{-6}$ mbar. The CDA model was dimensionally identical to the flight instrument but possessed a representative, rhodium chemical analyser target. The laboratory version used here had an accelerating grid support above the target, whereas the flight model has a self-supporting grid, although the composition of the target and the strength of the accelerating field are unchanged. This effectively simulated the impacts expected in flight, enabling the plasma dynamics to be investigated.

An impact event in the detector was triggered by signals read from the CDA model on standard oscilloscopes. The particle velocity $(v)$ and charge $(q)$ for each individual event were determined through detection along the accelerator beam line via induced charges on conducting cylinders arranged in a time-of-flight detector system (Burchell et al. 1999a). The mass $(m)$ for each particle is determined from Eq. (1), where the acceleration voltage $(V)$ was recorded for each event:

$m=(2 q V) / v^{2}$.

Figure 2 shows a schematic of the CDA instrument and a typical signal response to a hypervelocity impact on the centrally located chemical analyser target of the CDA. The resulting plasma was subject to a high electric field due to a $1 \mathrm{kV}$ potential difference maintained over the $3 \mathrm{~mm}$ separation between the accelerating grid and the rhodium target surface. This served to collect the negative plasma component on the target surface (QC) and accelerate the positive plasma component towards the centrally located detector (QM) beyond a 

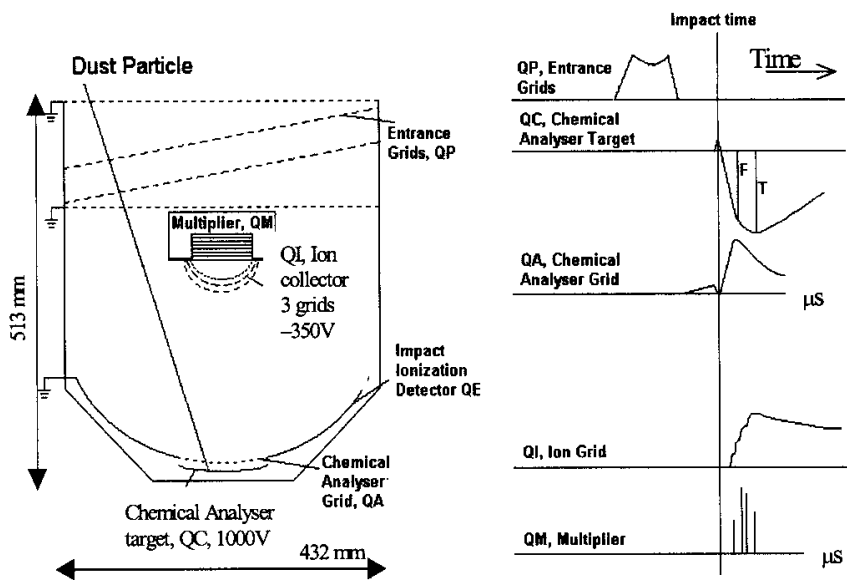

Fig. 2. Schematic of Cassini Cosmic Dust Analyser and typical signal responses to an impact on the chemical analyser target. An incident dust particle will (if charged) induce a signal QP on the entrance grids. If the particle impacts the Chemical Analyser target a charge pulse QC is observed on the target with a corresponding signal QA on the target grid. The ions produced in the impact drift to the ion detector where a signal QI is produced on the entrance grids and a final signal QM (i.e. the mass spectra) is produced by the ion multiplier.

drift region. Note that the QC signal has a fast rising component (whose magnitude is shown as $\mathrm{F}$ in Fig. 2) and a total magnitude ( $T$ in Fig. 2). In the subsequent analysis when ionization yield is referred to the magnitude was taken as the total magnitude $(T)$ recorded on target surface (QC). A small potential of $-350 \mathrm{~V}$ on the ion collection grids further assists in focusing the anions towards the ion multiplier. The passage across the drift region results in a time-of-flight spectrum containing those positively ionised components from the impactor and the target, thus the heavier components take longer to travel and appear later in the spectrum. For any single event the time-of-flight spectrum can be converted into a mass spectrum (atomic mass units) by using, for example, the relatively heavy rhodium (103 amu) constituent known to originate from the target material and the impact time taken from the target signal. The analysis assumed that each ion was singly charged. During flight, the target signal provides constraining information on the impacting microparticle, such as impact speed from the signal rise time (see later). For a more detailed description see Srama \& Grün (1996) and Srama et al. (2003).

\subsection{Organic-based Microprojectiles}

Traditionally the samples available for acceleration by van de Graaff were limited; they have to be electrically conducting, easily chargeable and be readily available in powdered form. Thus metals such as iron, or metal-coated particles, were used in the vast majority of impact ionization studies. However, certain other non-metallic materials also satisfy these criteria, and in 1990 Keaton and co-workers (Keaton et al. 1990) reported the first successful test-bench acceleration of conducting polymer-based particles. A number of conducting polymers, e.g. polypyrrole and polyaniline, are suitable for electrostatic acceleration; their conductivity is due to their

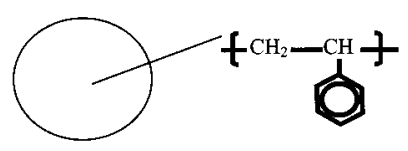
aqueous media
Polystyrene latex in

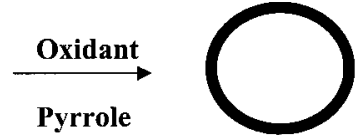

Polypyrrole coated latex particle
Fig. 3. Schematic of the synthesis of a polypyrrole coated polystyrene latex sample. The latex is initially in solution, the polypyrrole and an oxidant are added and the resultant particles have the desired coating.

highly conjugated backbones and the mobile charge carriers on those backbones. In addition, recent synthetic advances have enabled electrically insulating particles such as polystyrene latex to be coated in a thin, uniform conducting polymer overlayer (for example see Lascelles \& Armes 1995, 1997). Burchell et al. (1999b) reported the suitability of such coated latexes for electrostatic acceleration and found that they made excellent organic-based microparticles for use in impact ionization studies. A schematic representation of the synthetic procedure for adding a conducting polymer overlayer to a core particle (polystyrene) is shown in Fig. 3. Essentially an oxidant is added to an aqueous solution that contains sterically stabilised polystyrene latex. The monomer of choice, in this case pyrrole, is then added, and polymerisation ensues. The conducting polymer that is formed is insoluble in water and preferentially deposits onto the latex particles, producing a well-defined coreshell morphology. Further details of the synthesis, composition and detailed surface characterisation of such particles are given in Lascelles \& Armes (1997), and Khan \& Armes (2000).

There are several materials suitable for coating and, conveniently, these are often readily doped with specific elements of particular interest with regard to spectral investigations. This has greatly increased the range of chemical compositions available for acceleration. In addition, the synthesis provides samples with strictly controllable parameters. The mean particle diameter, for example, can be controlled and typically possesses a relatively narrow distribution. This is apparent in Fig. 4, an electron micrograph of a $100 \mathrm{~nm}$ diameter polypyrrole latex (sample PP). In addition, when core-shell particles are used, the desired size of the core can be chosen in advance and be prepared with a narrow size distribution, again ensuring nearmonodisperse microparticles. Each sample is typically accelerated to a narrow velocity range that is determined by the particle size (see Burchell et al. 1999b; Burchell et al. 2002). Thus samples of different sizes enable a range of velocities to be investigated thoroughly, while maintaining the constant chemical composition that is particularly useful for spectral investigations. It has been shown in preliminary work that these particles can be used in laboratory-based impact ionization experiments that incorporate time-of-flight mass spectrometry. Goldsworthy et al. (2002) showed that the particles can yield mass spectra (in a Cassini CDA type detector), and Kissel \& Krueger (2001) showed that mass spectra can be obtained from an instrument similar to that used on the Stardust space mission.

The relatively low densities (ca. 1080-1180 $\mathrm{kg} \mathrm{m}^{-3}$ ) of these new polypyrrole-coated polystyrene microparticles 
Table 1. Summary of the characteristics of the polypyrrole-based microparticles. ${ }^{1}$ The mean diameter was determined using a Brookhaven disc centrifuge photosedimentometry (described by Lascelles \& Armes 1997). ${ }^{2}$ Densities for the PP and PP3 samples were obtained from the known densities of the components and the chemical composition, assuming additivity. Other values were determined by helium pycnometry.

\begin{tabular}{clllll}
\hline \hline Sample & Description & $\begin{array}{l}\text { Mean } \\
(\mu \mathrm{m})\end{array}$ & diameter. $^{1}$ & $\begin{array}{l}\text { Mean Density } \\
\left(\mathrm{g} \mathrm{cm}^{-3}\right)\end{array}$ & $\begin{array}{l}\text { Mean } \\
\text { Mass }(\mathrm{kg})\end{array}$ \\
\hline PP & Pure polypyrrole & 0.10 & 1.46 & $7.64 \times 10^{-19}$ \\
PP3 & $\begin{array}{l}\text { Large polypyrrole- } \\
\text { coated polystyrene }\end{array}$ & 1.57 & 1.08 & $2.19 \times 10^{-15}$ \\
PP4 & $\begin{array}{l}\text { Medium polypyrrole- } \\
\text { coated polystyrene }\end{array}$ & 0.48 & 1.18 & $6.83 \times 10^{-17}$ \\
PP5 & $\begin{array}{l}\text { Small polypyrrole- } \\
\text { coated polystyrene }\end{array}$ & 0.19 & 1.16 & $4.17 \times 10^{-18}$ \\
& $\begin{array}{l}\text { Polypyrrole-coated } \\
\text { aluminosilicate clay }\end{array}$ & $\begin{array}{l}\text { Sphere } \\
0.82\end{array}$ & equivalent & 2.54 & Polydisperse \\
\hline
\end{tabular}

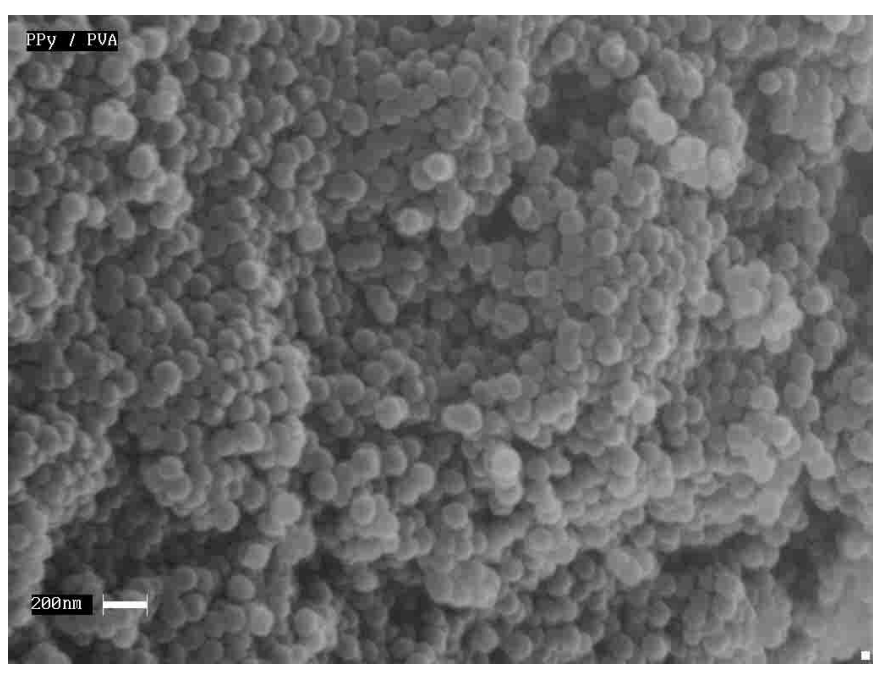

Fig. 4. Electron micrograph of close packed polypyrrole particles (100 nm diameter, sample PP). Scale bar represents $200 \mathrm{~nm}$. Note the high degree of uniformity in size of the particles.

provide a good comparison to traditionally accelerated iron (density $7800 \mathrm{~kg} \mathrm{~m}^{-3}$ ). Furthermore, their chemical compositions (up to $90 \%$ carbon by mass) mimic the carbon-rich compositions of cosmic dust grains, although it should be noted that at least some cosmic grains are believed to be highly porous (the highly porous chondritic-porous microparticles identified by Bradley et al. 1983), whereas these microparticles are nonporous in nature. In addition, it should also be noted that the bonding states of the atoms can also influence the ionization yield. Therefore complete equivalence to cosmic particles cannot be established.

In this work five types of microparticles are studied, four of which rely on a thin conducting overlayer of polypyrrole on an organic (polystyrene latex) or inorganic (clay) core material and the fifth projectile comprised around $90 \%$ polypyrrole by mass. This last polypyrrole sample (labelled PP) is shown in Fig. 4. Of the four microparticles with core-shell morphologies, three samples have the same core composition but different mean diameters $(0.19,0.48$, and $1.57 \mu \mathrm{m}$, named PP3, PP4 and PP5 respectively), consisting of a polystyrene (PS) latex core with a thin overlayer of polypyrrole. This enables the systematic investigation of the effect of varying the impact velocity on the spectral response. The fourth sample of coreshell microparticle consists of a core of aluminosilicate clay coated with a polypyrrole shell (labelled AlSi). This latter sample possessed a polydisperse platelet morphology, rather than near-monodisperse spherical particles, this is due to the natural origin of the clay core material.

The synthesis of the polypyrrole particles (sample PP) is described in detail in Armes \& Vincent (1987) and Armes et al. $(1987,1991)$. The synthesis of the polypyrrole-coated polystyrene latex particles is described in Lascelles \& Armes (1995, 1997), Khan \& Armes (2000) and Khan (2001). The synthesis of the polypyrrole-coated aluminosilicate particles has not been reported previously and is described in the Appendix.

These five projectiles thus include three distinctly different compositions for mass spectral comparison; two different organic compositions and a mineral-based composition. In addition, mass spectra due to impacts of iron microparticles were also obtained. Table 1 summarises the key characteristics (mean measured particle diameter and density and the corresponding derived mean mass) of each type of microparticle. Ideally, polypyrrole has the approximate elemental composition of $\mathrm{C}_{4} \mathrm{H}_{3} \mathrm{~N}_{1} \mathrm{Cl}_{0.33}$ (normalised to nitrogen). In practice, however, it contains an additional oxygen component due to partial oxidation of the polymer backbone. Typically for these core-shell particles the conducting polymer coating represents less than $10 \%$ by mass, therefore the overall chemical compositions are dominated by the core materials, in this case polystyrene latex (ideally $\mathrm{C}_{8} \mathrm{H}_{8}$ ) or aluminosilicate clay (ideally $\mathrm{Na}_{2} \mathrm{Al}_{2} \mathrm{SiO}_{6}$ ).

\section{Results}

Each sample was successfully charged and electrostatically accelerated using a 2 MV van de Graaff accelerator. Figure 5 shows the mass-velocity data obtained for each sample, where the mass has been calculated using Eq. (1). In the data the velocity is measured with an accuracy of typically better than $1 \%$ 
Table 2. Response of dust samples on acceleration, ${ }^{1}$ true population defined by mass analysis, ${ }^{2}$ mode diameter from the Gaussian fit of true population diameter distribution (standard deviation).

\begin{tabular}{|c|c|c|c|c|c|c|c|}
\hline Sample & Description & $\begin{array}{l}\text { Total } \\
\text { No. of } \\
\text { events }\end{array}$ & $\begin{array}{l}\text { Velocity } \\
\text { response } \\
\mathrm{km} \mathrm{s}^{-1}\end{array}$ & $\begin{array}{l}\text { No. of } \\
\text { "true" } \\
\text { events } 1\end{array}$ & $\begin{array}{l}\text { Velocity } \\
\text { Response } \\
\text { "true" particles }{ }^{1} \\
\mathrm{~km} \mathrm{~s}^{-1}\end{array}$ & $\begin{array}{l}\text { Diameter of "true" } \\
\text { particles }^{2} \\
\mu \mathrm{m}(\sigma)\end{array}$ & $\begin{array}{l}\text { Mass of "true" } \\
\text { particles, kg }\end{array}$ \\
\hline PP & Pure polypyrrole & 45 & $5.1-56.9$ & 32 & $16.3-39.3$ & $0.13 \pm 0.03$ & $1.68 \times 10^{-18}$ \\
\hline PP3 & $\begin{array}{l}\text { Large polypyrrole- } \\
\text { coated polystyrene }\end{array}$ & 82 & $3.2-15.6$ & 43 & $3.2-6.3$ & $1.55 \pm 0.10$ & $2.11 \times 10^{-15}$ \\
\hline PP4 & $\begin{array}{l}\text { Medium } \\
\text { polypyrrole-coated } \\
\text { polystyrene }\end{array}$ & 123 & $5.7-38.9$ & 83 & $5.7-17.6$ & $0.35 \pm 0.24$ & $2.60 \times 10^{-17}$ \\
\hline PP5 & $\begin{array}{l}\text { Small polypyrrole- } \\
\text { coated polystyrene }\end{array}$ & 36 & $3.9-26.2$ & 27 & $10.1-26.2$ & $0.24 \pm 0.02$ & $8.40 \times 10^{-18}$ \\
\hline ALSI & $\begin{array}{l}\text { Polypyrrole-coated } \\
\text { aluminosilicate clay }\end{array}$ & 80 & $3.4-50.7$ & 80 & $3.4-50.7$ & Polydisperse & $10^{-19}-10^{-15}$ \\
\hline
\end{tabular}

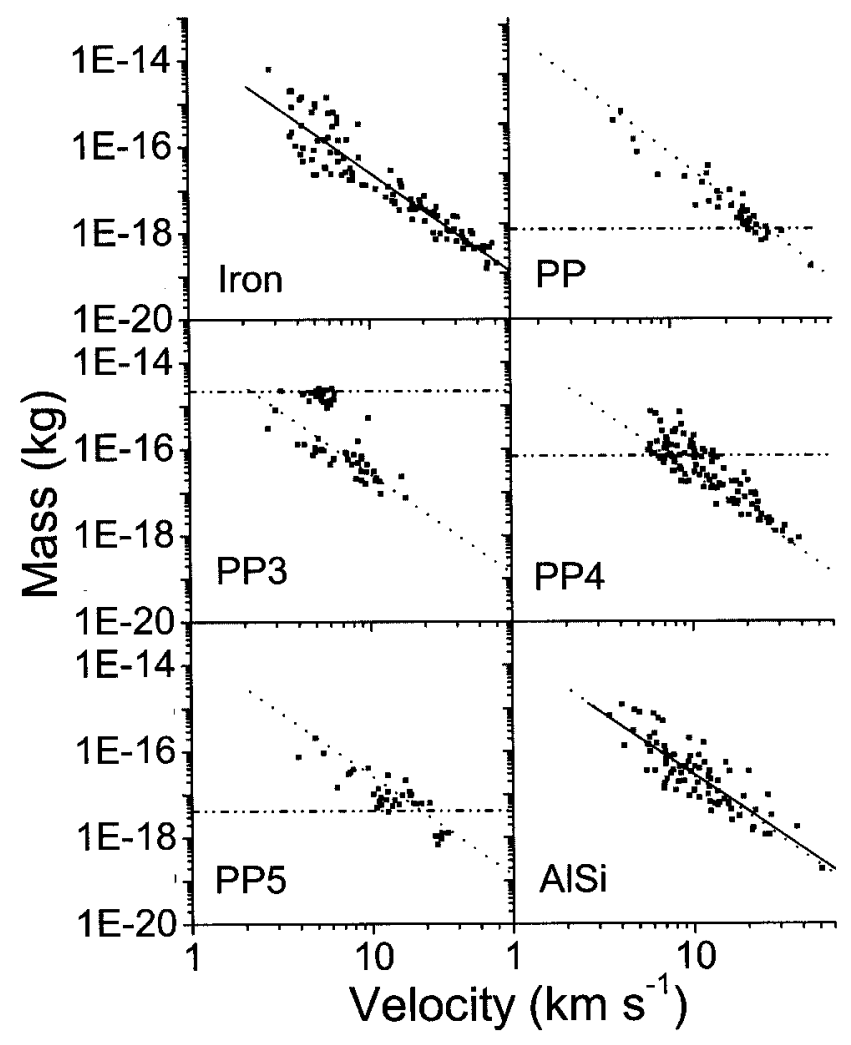

Fig. 5. Mass vs. velocity relationship for accelerated microparticles. The iron best fit is indicated on subsequent plots as a dotted line. The horizontal dashed lines indicate the sample mean mass for each sample as quoted in Table 1 .

at low speeds $\left(1 \mathrm{~km} \mathrm{~s}^{-1}\right)$, rising to $5 \%$ at $50 \mathrm{~km} \mathrm{~s}^{-1}$. The mass is accurate to $2 \%$ at low speed, rising to $10 \%$ at $50 \mathrm{~km} \mathrm{~s}^{-1}$. See Burchell et al. (1999a) for more details. An iron sample is also shown for comparison, the best fit for which $(m=(2.5 \pm 0.6) \times$ $10^{-14} v^{-(2.8 \pm 0.2)}, r=-0.93$, units of $\mathrm{kg}$ and $\mathrm{km} \mathrm{s}^{-1}$, where $r$ is the regression coefficient for the fit) is shown on subsequent sub-plots in Fig. 5 as a dotted line. The horizontal dashed lines indicate the mean mass of each sample obtained during synthesis and quoted in Table 1. Table 2 summarises the velocity response obtained with each sample.

In Fig. 5, the iron microparticles display a characteristically broad particle size distribution with a correspondingly wide velocity response. For the four conducting polymer-based microparticles (PP, PP3, PP4 and PP5) the mean mass expected from synthesis coincides fairly well with the mode measured mass. The mono-disperse nature of the samples from synthesis is shown in Fig. 2 and similar figures in the various references (e.g. Burchell et al. 1999b, 2002). However, PP4 exhibits a somewhat broader size distribution than expected and, in the case of PP5, the expected mean mass differs slightly from that observed. The AlSi sample has the expected polydisperse mass distribution, with a fit $m=(1.9 \pm 0.9) \times 10^{-14} v^{-(2.8 \pm 0.2)}, r=$ -0.84 (units of $\mathrm{kg}$ and $\mathrm{km} \mathrm{s}^{-1}$, where $r$ is the regression coefficient for the fit). This is shown as a solid line on the data.

Further, it is apparent that within some samples there are a number of events with masses that are significantly different from the quoted mean value. Samples PP, PP4 and PP5 have a population of events with more massive particles. These are believed to be examples of where individual particles have clumped together to form larger agglomerates. Samples PP3, PP4 and PP5 also display populations with smaller particles, possibly due to particle fragmentation as a result of the high surface charge used for acceleration. As stated, the modal mass for sample PP5 appears slightly larger than the quoted value obtained for the aqueous dispersion by disc centrifuge photosedimentrometry. Burchell et al. (1999b) also accelerated particles from this sample,and reported a slightly higher mass than the expected value, in line with that observed here. In the PP, PP3, PP4 and PP5 samples, these high and low mass populations have been removed from further analysis to ensure the composition is accurately known for spectral comparison purposes. The "true" velocity range of the remaining events is quoted in Table 2 along with the calculated modal diameter and mass. The AlSi sample has no well-defined mode mass and thus the data over the full velocity range is used in all the work.

Generally the results of charging and acceleration from these new low-density projectiles were as expected. 
Table 3. Results of fits to the data for the target yield normalised to mass $\left(\mathrm{C} \mathrm{kg}^{-1}\right)$ vs. velocity $\left(\mathrm{km} \mathrm{s}^{-1}\right)$ (Fig. 6), where $r$ is the regression coefficient.

\begin{tabular}{|c|c|c|}
\hline Projectile & Fit & $r$ \\
\hline Iron & $(7.8 \pm 1.6) \times 10^{-1} v^{(3.36 \pm 0.08)}$ & 0.97 \\
\hline Iron $v<15 \mathrm{~km} \mathrm{~s}^{-1}$ & $(1.4 \pm 0.7) v^{(3.1 \pm 0.2)}$ & 0.91 \\
\hline Iron $v>15 \mathrm{~km} \mathrm{~s}^{-1}$ & $(3.9 \pm 2.6) \times 10^{-2} v^{(4.3 \pm 0.3)}$ & 0.89 \\
\hline $\begin{array}{l}\text { Polypyrrole-coated polystyrene (PP3, } \\
\text { PP4, PP5 samples combined) }\end{array}$ & $(1.7 \pm 0.4) v^{(2.89 \pm 0.09)}$ & 0.94 \\
\hline Polypyrrole (PP sample) & $(1.6 \pm 1.0) \times 10^{-3} v^{(5.2 \pm 0.3)}$ & 0.95 \\
\hline $\begin{array}{l}\text { Polypyrrole-coated alumininosilicate clay } \\
\text { (AlSi sample) }\end{array}$ & $(2.4 \pm 0.5) v^{(2.9 \pm 0.1)}$ & 0.94 \\
\hline
\end{tabular}

The PP sample was accelerated in the range 16 to $39 \mathrm{~km} \mathrm{~s}^{-1}$. The PPY-coated PS samples (PP3, PP4 and PP5) cover a velocity range from 3 to $26 \mathrm{~km} \mathrm{~s}^{-1}$. Finally, because it has a broad mass distribution, the AlSi sample could be accelerated from 3 to $50 \mathrm{~km} \mathrm{~s}^{-1}$. It is thus possible to study detector response properties and the impact mass spectra over a wide range of impact speeds.

\subsection{Target response}

These low-density samples enabled the target response to be assessed when impacted with materials of significantly different densities compared to the standard iron microparticles. Figure 6 shows the negative plasma components for impacts on the rhodium chemical target (QC) yield, normalised to the mass of each impactor. The uncertainty in the normalised ionization yield is typically $10 \%$ at low speeds, rising to some $15 \%$ at $50 \mathrm{~km} \mathrm{~s}^{-1}$. The results of the fits to Fig. 6 are given in Table 3, only the "true" particles as determined by the mass analysis are included in the data. A power law fit is given as is standard with this type of data (i.e. the data cover several orders of magnitude).

Figure 6a shows the iron data set and the fit that is shown (solid line) is also given in Figs. $6 \mathrm{~b}$ and $6 \mathrm{c}$ as a dashed line for comparison. The data for the purely organic microparticles are shown in Fig. 6b. Since the PP3, 4 and 5 samples have similar compositions it seems sensible to fit these data with a single function (the solid line at velocities of $2-30 \mathrm{~km} \mathrm{~s}^{-1}$ ). At higher velocities in Fig. $6 \mathrm{~b}$ are shown the data for the PP sample, which is fit separately (the solid line in the range $15-45 \mathrm{~km} \mathrm{~s}^{-1}$ ). The PP data have a greater dependence on impact speed, hence an increased power of $v$ in the fit given in Table 3. The transition in Fig. $6 \mathrm{~b}$ to the steeper slope in velocity occurs at approximately $20 \mathrm{~km} \mathrm{~s}^{-1}$. To test if this is somehow independent of the nature of the projectile, the iron data in Fig. 6a were fitted again, this time in two separate ranges of velocity (greater than and less than $15 \mathrm{~km} \mathrm{~s}^{-1}$ ). The results are also given in Table 3. There is an apparent effect, with an increased power of $v$ seen in the higher speed data set. Note that the speed at which the slope changes is lower for iron $\left(15 \mathrm{~km} \mathrm{~s}^{-1}\right)$ than for the organic particles $\left(20 \mathrm{~km} \mathrm{~s}^{-1}\right)$. It is also possible to separate the iron data into three speed regimes, low speed, intermediate speed and high speed. This was done for

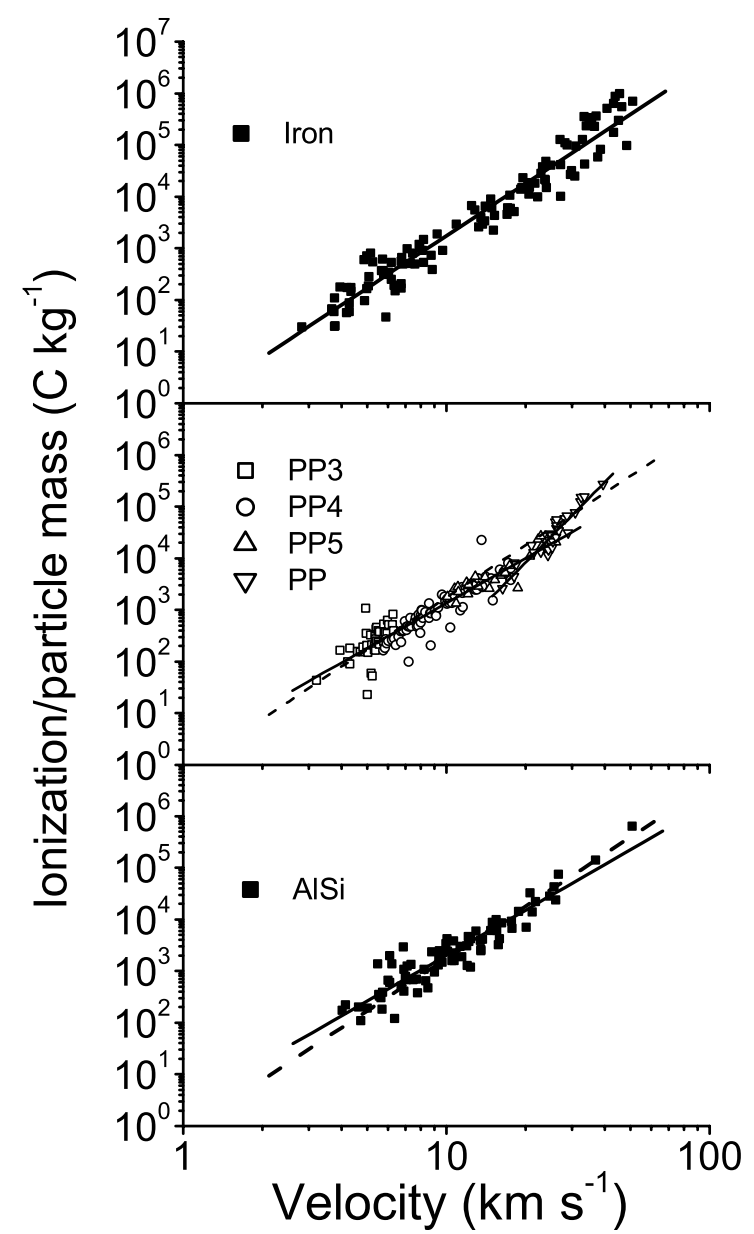

Fig. 6. Target ionization charge normalised to particle mass vs. velocity for a) iron, b) organic and c) mineral containing microparticles. The fit to the iron data is indicated on subsequent plots as a dashed line. The data for each fit are given in Table 3. In b) the samples PP3, PP4 and PP5 were fitted as a combined set, with PP fitted separately.

iron particle impacts on a rhodium target for example in Stübig et al. (2003). Their data for ionization charge/mass vs. impact speed are very similar in magnitude to those presented here. At high speed they find the a fit which has $v^{4.4}$, in good agreement with the value of $4.3 \pm 0.3$ found here (Table 3 ). At lower speeds they divide the data into two speed regimes. At the lowest speeds $\left(<4 \mathrm{~km} \mathrm{~s}^{-1}\right)$ they find $v^{3.6}$, and at intermediate speeds 
Table 4. Results of fits to the data for the target rise time (s) vs. velocity $\left(\mathrm{km} \mathrm{s}^{-1}\right)$ (Fig. 7).

\begin{tabular}{ll}
\hline \hline Projectile material & Fit \\
\hline Iron & $3.8 \times 10^{-6}-1.3 \times 10^{-7} v$ \\
Samples PP3, PP4, PP5, PP combined & $3.0 \times 10^{-6}-1.1 \times 10^{-7} v$ \\
Sample AlSi & $4.5 \times 10^{-6}-2.65 \times 10^{-7} v$ \\
\hline
\end{tabular}

they find $v^{2.6}$. Here the data are fit with a single power law at low and intermediate speeds and we obtain $v^{(3.1 \pm 0.2)}$, intermediate between, and compatible with, the two values of Stübig et al. (2003). Finally, Fig. 6c shows the data for the AlSi sample with the corresponding fit (solid line).

Within the scatter of the data, the normalised target yield from all the low-density projectiles agrees with that obtained for iron, despite the large density differences and the varying chemical compositions. Even the changing behaviour at low and high velocities is reproducible. Thus for this type of target material (rhodium) there is little, if any, difference in impact ionization yield that is related to anything other than particle mass and velocity, i.e. it is independent of projectile composition for representatives of three widely varying classes of particles (metals, organics and silicates).

Charge signals recorded from the target can also be used to provide an indication of the impact speed of the impinging microparticle. This is important, because in space the speed of the impactors is usually unknown. There are exceptions to this. The CDA detector carries charge-sensitive grids across its entrance, which can be used to find a transit time (and hence speed) for heavily charged particles entering the detector. For the Stardust mission the encounter speed with the comet dominates the impact speed for dust particles emitted from the nucleus, thus giving an impact speed of $6.1 \mathrm{~km} \mathrm{~s}^{-1}$ with only a narrow spread in speed. However, in general a measure of impact speed from the impact itself is clearly desirable. In CDA (Fig. 2) the signal recorded from the target (QC or QE) for an individual event provides an indication of the impact velocity. In the laboratory this is easily verified through independent particle detection in the beam line of the accelerator. The time required for the chemical target charge signal (QC) to reach a maximum is shown in Fig. 7 and is measured with an accuracy of order $50 \mathrm{~ns}$. The iron data are shown in Fig. 7a, the data for the PP and PP3, 4 and 5 samples are shown together in Fig. 7b, and the AlSi data are depicted in Fig. 7c. In each case most of the data display common behaviour. At low speeds (i.e. a few $\mathrm{km} \mathrm{s}^{-1}$ ) there is a limiting maximum rise time of $(3.75 \pm 0.75) \mu \mathrm{s}$. This decreases linearly as the impact speed increases. Finally, above $20-30 \mathrm{~km} \mathrm{~s}^{-1}$ there appears to be a minimum rise time of $(0.20 \pm 0.05) \mu$ s that is independent of the impact speed. Indeed, for the AlSi sample this minimum rise time is observed even in some of the lower speed data. It is not clear what causes this behaviour, but it is observed in data for all three types of projectile, i.e. metal (iron), organic (the polypyrrole, $\mathrm{PP}$, and polypyrrole-coated polystyrene samples, PP3, PP4 and PP5) and mineral (the aluminosilicate sample, $\mathrm{AlSi})$.

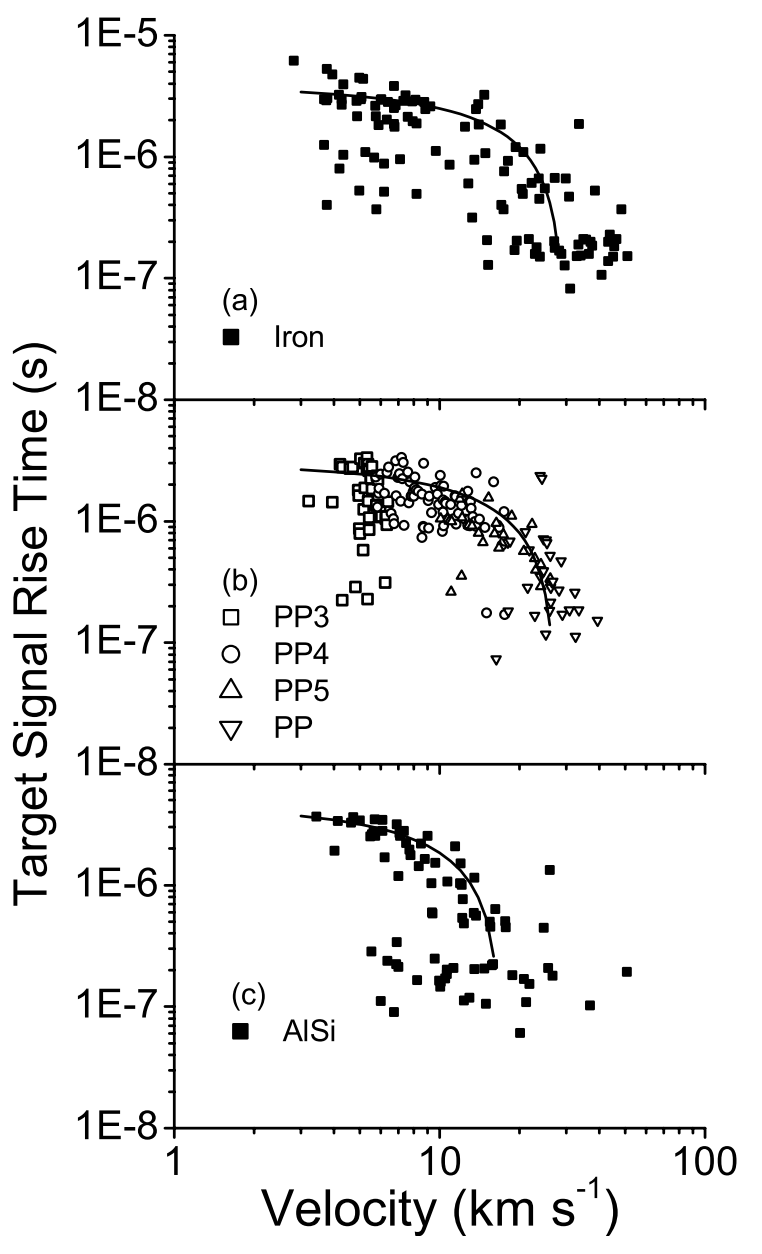

Fig. 7. The target charge signal rise time vs. velocity for a) iron, b) organic and c) mineral containing microparticles. The data for the fit curves are given in Table 4.

Fits are shown to the linear part of the data in Fig. 7 with the results given in Table 4 (in Fig. 7 the fit lines do not appear as straight lines because of the log-log axes of the graph). Note that no goodness of fit criteria or uncertainties on the fit coefficients are given for the fits in Table 4. This is because the most obvious outlying data points were excluded in each case in order to obtain the fits. This effectively forces a fit with an artificially high goodness of fit value. However, it is a justified procedure because it reveals the characteristics of the majority of the data. Several features emerge: While the fits to each data set (metal, organic and mineral) are linear in each case, there are differences between the various projectiles. The iron and the organic-based microparticles behave similarly, but the fit for AlSi has a much greater slope. This suggests that the 
critical impact speed above which the linear behaviour ceases to be applicable depends on the composition of projectile. It is $16 \mathrm{~km} \mathrm{~s}^{-1}$ for the mineral-based (AlSi) particles and $28 \mathrm{~km} \mathrm{~s}^{-1}$ for both the iron and organic-based (PP and PP3, PP4 and PP5) microparticles. A single type of behaviour is thus found for the ionization yield that is independent of the nature of the impactor in the laboratory, but not for the signal rise time and thus the determination of impact speed. On a spacecraft-deployed instrument it is this combination of impact speed and ionization yield measurements that permits the determination of the mass of the impacting particle. It is thus important to know if the instrument response depends on the nature of the impactor and its speed. The results indicate that the laboratory calibrations for the chemical target in CDA-type instruments are sensitive to particle composition. However, it should be noted that the data for the calibration of impact speed from signal rise time has a large scatter and hence there is a large uncertainty in calculating the impact speed; this problem is exacerbated when the impacting micrometeoroid composition and density are unknown. In addition, high impact speeds cannot be determined with the current laboratory instrument.

\subsection{Spectral response}

There is a large amount of information contained within the spectral data collected by the time-of-flight system. In Fig. 8 typical spectra are shown for impacts of iron at differing impact speeds (with a linear vertical scale). The conversion from time to mass on the horizontal axis in Fig. 8 was achieved using the relation

$\Delta t=D \sqrt{ } \Delta m$

The coefficient $D$ in the mass scale for each event has been fitted individually using two peaks, sodium (23 amu) and potassium (39 amu), and the mass scale is then verified by comparison with the impact time given by the target signal. As contaminants within the system, sodium and potassium are always present in the spectra at these velocities and are generally easy to identify. It is found that $D$ typically has the value of $(5.25 \pm 0.08) \times 10^{-7}$ (where $t$ is in s and $m$ in amu).

Populations of different ion species and their evolution with impact speed can be readily seen. A discussion of this is given below. Figure 9 shows typical spectra for samples AlSi, PP and PP5. In Fig. 10 summed mass spectra are shown for the PP3 and PP4 samples. A summed spectrum is one where all the individual mass spectra of the "true" particle impacts within a population of microparticles have been added together and divided by the number of "true" impacts. Again in Fig. 10 it is possible to see an evolution between PP3 (average $\sim 5 \mathrm{~km} \mathrm{~s}^{-1}$ ) and PP4 (average $\sim 9 \mathrm{~km} \mathrm{~s}^{-1}$ ) with increasing impact speed. To further display this dependence on impact speed, the data have been reduced to the graphical representation shown in Figs. 11 to 13 . Here event impact velocity $\left(\mathrm{km} \mathrm{s}^{-1}\right)$ is plotted along the vertical axis and mass (atomic mass units) along the horizontal axis. Therefore, each horizontal line of dots represents a single impact event. Each dot represents a peak in the mass spectrum, with the size reflecting the prominence of that peak within the event. The horizontal bars give the width of each
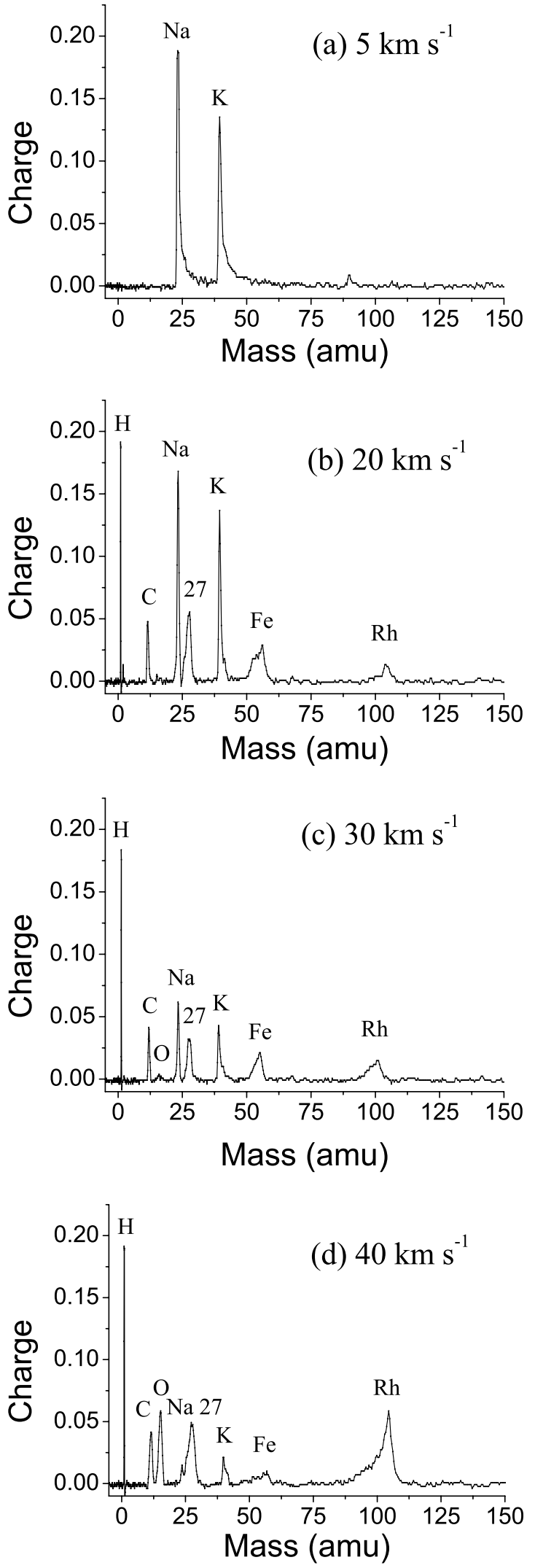

Fig. 8. Typical mass spectra for impacts of iron onto rhodium a) $\sim 5 \mathrm{~km} \mathrm{~s}^{-1}$, b) $\sim 20 \mathrm{~km} \mathrm{~s}^{-1}$, c) $\sim 30 \mathrm{~km} \mathrm{~s}^{-1}$, d) $\sim 40 \mathrm{~km} \mathrm{~s}^{-1}$.

peak, providing an indication of their separation (if not shown, the width was less than the size of the symbol used). Care must 

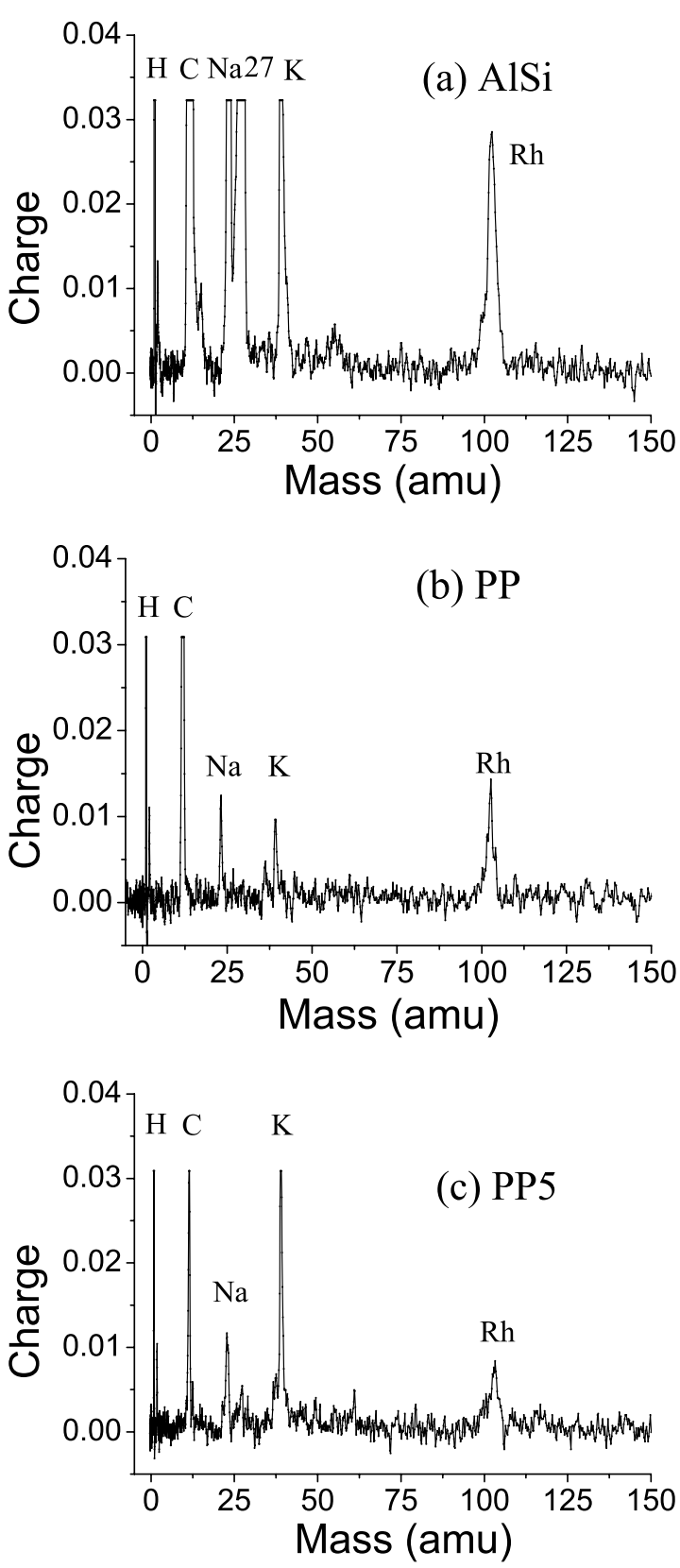

Fig. 9. Typical mass spectra for impacts of various projectiles onto rhodium, a) AlSi (mineral containing) $\sim 27.0 \mathrm{~km} \mathrm{~s}^{-1}$, b) PP (pure polypyyrole) $\sim 26.8 \mathrm{~km} \mathrm{~s}^{-1}$, c) PP5 (polypyrrole coated polystyrene latex) $\sim 24.1 \mathrm{~km} \mathrm{~s}^{-1}$.

be taken when comparing projectile samples in Figs. 11-13 as the ranges of impact speed overlap between the samples. Displayed in Fig. 11 is the spectral response from iron projectiles. It shows a response that has been commonly reported (see for example Ratcliff et al. 1997). At low velocities the alkali contaminants sodium (23 amu) and potassium (39 amu) dominate the spectra, gradually decreasing in relative abundance as the velocity increases. The projectile (iron, $56 \mathrm{amu}$ ) and target (rhodium, $103 \mathrm{amu}$ ) materials are not always clearly present, particularly at low velocities. In events with impact speeds below $15 \mathrm{~km} \mathrm{~s}^{-1}$ iron is only apparent in approximately $22 \%$ of events, but above $15 \mathrm{~km} \mathrm{~s}^{-1}$ this rises to approximately $82 \%$.

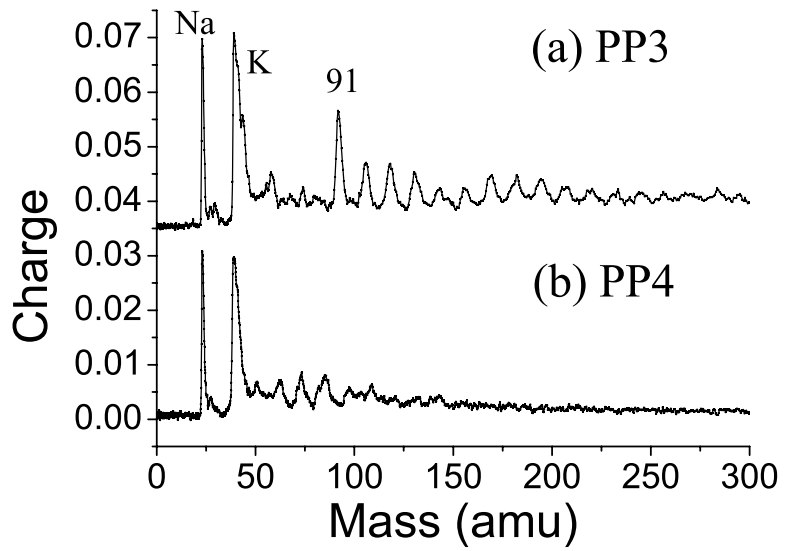

Fig. 10. Summed mass spectra for impacts of organic microparticles onto rhodium. a) Sample PP3 $\left(\sim 5 \mathrm{~km} \mathrm{~s}^{-1}\right)$ and b) sample PP4 $\left(\sim 9 \mathrm{~km} \mathrm{~s}^{-1}\right)$.

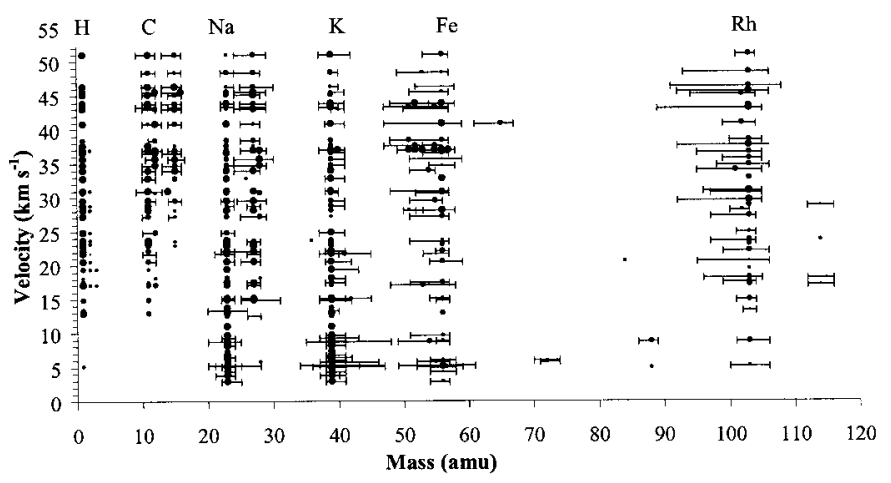

Fig. 11. Graphical representation of spectra taken with a CDA type instrument for impact of iron projectiles. Each horizontal line of dots represents an individual particle impact event with velocity indicated by the $y$-axis. Each dot represents a spectral peak with the size indicating the relative prominence of each species and the error bar signifies the peak width.

The iron and rhodium do, however, become relatively more abundant as the velocity increases. In addition, the peak response broadens, with an asymmetric shape favouring lower mass (earlier arrival times of ions), possibly due to a larger portion of the ions possessing higher initial energies. There are a number of other species that are believed to be contaminants. On passing a velocity threshold of approximately $12 \mathrm{~km} \mathrm{~s}^{-1}$, hydrogen (1 amu), carbon (12 amu) and a species at $27 \mathrm{amu}$ become regularly apparent and proceed to become more abundant as the velocity increases, unlike sodium and potassium whose relative abundance declines. The species at $27 \mathrm{amu}$ is suggestive of aluminium. However, this species is not expected and this signal is therefore believed to be a molecular component $\left(\mathrm{C}_{2} \mathrm{H}_{3}^{+}\right)$, perhaps originating from hydrocarbon-based oils within the van de Graaff system. This contamination is also expected to be the primary source of the hydrogen and carbon signals. Additionally, oxygen also becomes increasingly abundant at velocities of approximately $25 \mathrm{~km} \mathrm{~s}^{-1}$ and upward, possibly originating from oxidation of the projectile material. Another possible origin of the oxygen is from water absorbed onto the surface of the target. 


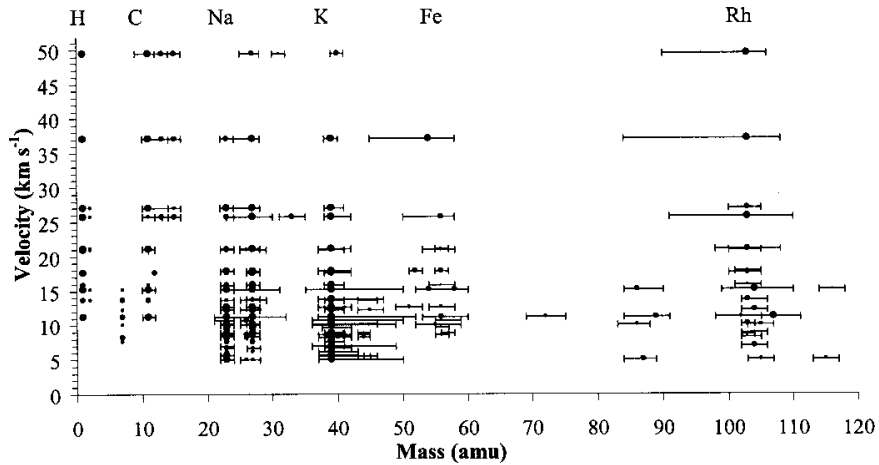

Fig. 12. Graphical representation of spectra taken with a CDA type instrument for impact of aluminosilicate-based projectiles (sample AlSi).
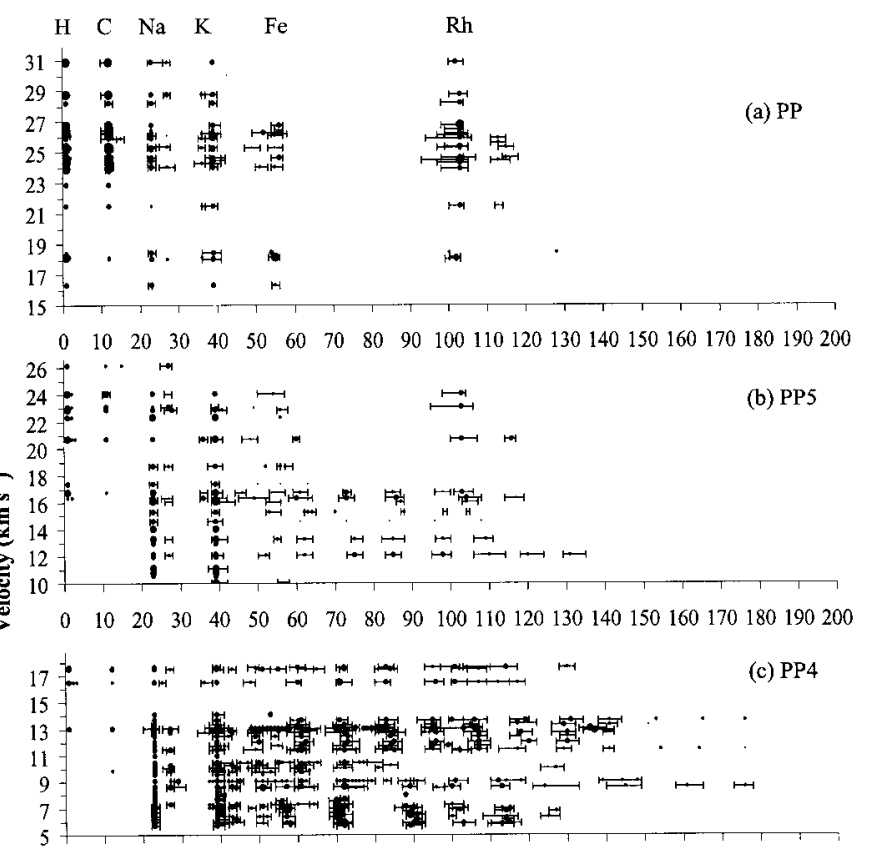

$\begin{array}{llllllllllll}0 & 10 & 20 & 30 & 40 & 50 & 60 & 70 & 80 & 90 & 100110120130140150160170180190200\end{array}$

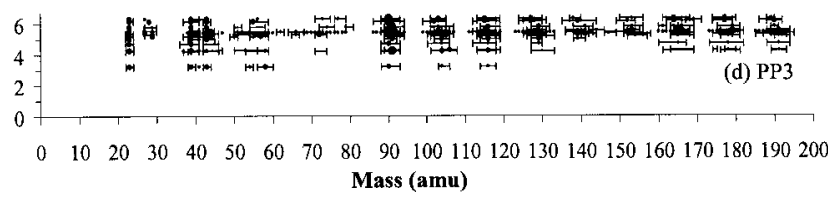

Fig. 13. Graphical representation of spectra taken with a CDA type instrument for impact of various polypyrrole and polypyrrole-coated polystyrene particle samples a) PP, b) PP5, c) PP4, d) PP3.

On occasion it has been observed in high velocity events that the iron peak becomes resolved into two distinct peaks of approximately equal magnitude, typically $53 \mathrm{amu}$ and $56 \mathrm{amu}$. Although iron has many isotopes spanning 54-59 amu that may give rise to a broad response, 56 amu is by far the most abundant $(\sim 92 \%)$. Thus an iron isotope effect does not explain this phenomenon. An alternative suggestion is that it represents the appearance of another meta-stable hydrocarbon molecule from the oil contamination, although why other such species were not also observed is not understood. It may be doubly ionised rhodium; although previous work has shown little evidence of doubly ionised species this should not be discounted. Another rare feature is a small signal at $\sim 115 \mathrm{amu}$, occurring in $\sim 3 \%$ of all iron events and at velocities in the range of $16-29 \mathrm{~km} \mathrm{~s}^{-1}$. It possibly represents a complex formed by the target material and carbon $\left(\mathrm{RhC}^{+}\right)$. Such species have been reported in the past (Knabe \& Krüger 1982). At low velocities, other features are occasionally observed at around $\sim 72$ amu and $\sim 87 \mathrm{amu}$.

Generally speaking the spectra are well behaved; that is, at a particular velocity the spectral response between events is typically the same, although the amplitudes of each species vary significantly (by a factor of two in extreme cases). Some ionic species become apparent only at high impact speed. Occasionally a species will appear at a velocity lower than expected and indeed sometimes is not observed at all. This behaviour for impacts of iron particles on rhodium is similar to that reported in earlier work (e.g. Ratcliff et al. 1997).

Displayed in Fig. 12 is the spectral response obtained from the aluminosilicate clay sample that contains a preponderance of sodium (ideal composition $\mathrm{Na}_{2} \mathrm{Al}_{2} \mathrm{SiO}_{6}$ ) coated in less than $10 \%$ polypyrrole (ideally $\mathrm{C}_{4} \mathrm{H}_{3} \mathrm{~N}_{1} \mathrm{Cl}_{0.33}$ ). The majority of these events are below $20 \mathrm{~km} \mathrm{~s}^{-1}$ but range up to $\sim 50 \mathrm{~km} \mathrm{~s}^{-1}$. At first glance these appear to be similar to the iron data set, although on closer inspection they are significantly different. As before, rhodium broadens to lower mass with increasing impact velocity and there is occasionally evidence of a feature at $115 \mathrm{amu}$ but this time at lower velocities $\left(5-15 \mathrm{~km} \mathrm{~s}^{-1}\right)$. The sodium and potassium contaminants again dominate at lower velocities but here sodium, as a component of the projectile, should remain prominent at high velocities. Unfortunately, there are only two suitably fast events to observe this and one does not indicate sodium. The significance of this should not be overstated since such events may be atypical and may possibly represent a polypyrrole fragment (which would contain no sodium). Despite forming a major component of the projectile, oxygen again appears only when the impact velocity is above $25 \mathrm{~km} \mathrm{~s}^{-1}$. Additionally, it is accompanied by nitrogen (14 amu), which is not observed in either the iron or the organic latex samples studied here. Nitrogen is not expected from the aluminosilicate core; it is present in the polypyrrole coating, but this introduces a puzzle. If the nitrogen originated from the polypyrrole, one would expect to have observed this signal in each of the PPY-containing microparticles at these velocities but this was not the case. Only the polypyrrole microparticles have comparable velocities (PP, shown in Fig. 13a) and this sample does not contain nitrogen. This suggests that the nitrogen signal originates from the aluminosilicate clay (and not the target for example), although this would require significant contamination of the clay by an unknown nitrogenbased compound. A particularly interesting feature is observed for impacts with hypervelocities between 7 and $16 \mathrm{~km} \mathrm{~s}^{-1}$ : a lithium signal (7 amu) occurs in approximately one third of these events. It is possible for lithium to be intercalated within the clay, yet no lithium signal is observed at higher speeds. Currently it is not possible to distinguish with confidence between silicon (28 amu), aluminium (27 amu) and the suspected hydrocarbon contamination (27 amu). However, a strong response is detected at $\sim 27 \mathrm{amu}$ at velocities of $5 \mathrm{~km} \mathrm{~s}^{-1}$, which is well below the minimum threshold $\left(\sim 15 \mathrm{~km} \mathrm{~s}^{-1}\right)$ for the hydrocarbon contaminant observed for iron events. There appears 
to be a species present at a mass of $\sim 44$ amu at impact velocities below $15 \mathrm{~km} \mathrm{~s}^{-1}$. There is also a species occasionally appearing within the mid 50's amu which could be due to the core material in the form of AlSi or due to the conducting coating. It is not believed to be due to iron contamination. At lower velocities there are again indications of features at $\sim 72 \mathrm{amu}$ and $\sim 87 \mathrm{amu}$.

Figure 13 shows the spectral response of the remaining four samples, first pure polypyrrole (PP) (a) followed by the various polypyrrole coated polystyrene samples PP5 (b), PP4 (c) and PP3 (d). Note that in Fig. 13 the various sub-plots have different ranges of velocity on the vertical scales. The PPY spectra (Fig. 13a) with an ideal composition of $\mathrm{C}_{4} \mathrm{H}_{3} \mathrm{~N}_{1} \mathrm{Cl}_{0.33}$ are relatively featureless in comparison to the other spectra presented in Fig. 13. However, its velocity range of $17-32 \mathrm{~km} \mathrm{~s}^{-1}$, is faster than most events in the PPY-PS samples. The usual species, hydrogen, carbon, sodium, potassium, and rhodium and, on occasion, a signal at $\sim 115$ amu are each present as expected in Fig. 13a. The 27 amu species is also present, although not at the same intensity as that found for iron events in the same velocity range. The absence of the pyrrole ring repeat unit (65 amu) suggests that the impact energy was sufficient to cause complete fragmentation of the polypyrrole chains into their constituent atoms. Hydrogen and carbon signals are indeed observed, as expected. However, it is interesting to note that nitrogen signals are not regularly observed, despite being seen at velocities exceeding $\sim 25 \mathrm{~km} \mathrm{~s}^{-1}$ in the AlSi sample. There are impact events here at speeds in excess of this. The lack of a nitrogen signal therefore suggests two things. First, the signal observed in the AlSi sample is from the clay and not from the conducting polymer. Second, some unknown mechanism prevents observation of the expected nitrogen signal. On this latter point it is noted that a $\mathrm{CN}^{-}$anion (amu 26) is always observed in the negative ion mode ToF-SIMS analysis of polypyrrole (Abel et al. 1994). If this negative ionic species is the major fate of the nitrogen atoms in the hypervelocity impact of PPY then it could account for the lack of a nitrogen signal in the positive ion spectra observed with this detector. There are species present at $\sim 56$ amu and sometimes at slightly lower masses. An unlikely possibility is that it originates from iron contamination of the target surface.

The PP5, PP4 and PP3 samples have almost identical compositions, but their different sizes provide a different velocity response after acceleration. If their spectral response is considered, it is immediately clear there is a strong velocity dependence. As the impact velocity increases the number of peaks decreases significantly, especially for higher mass signals. It is possible that the varying particle size influences the physical processes. However, even within each sample the spectrum changes with increasing velocity. The individual elements $\mathrm{H}$ and $\mathrm{C}$ do not begin to appear until above $\sim 12 \mathrm{~km} \mathrm{~s}^{-1}$, by which time $\mathrm{Na}$ and $\mathrm{K}$ have decreased in prominence, in agreement with observations made on other samples.

The PP3 sample, accelerated to the lowest speeds, exhibits a large number of peaks from $91 \mathrm{amu}$ up to $\sim 250 \mathrm{amu}$ (note the range above $200 \mathrm{amu}$ is not shown) with regular spacings of $\sim 12-14 \mathrm{amu}$. This indicates a large number of related molecular fragments, most likely differing by additional $\mathrm{CH}$ or $\mathrm{CH}_{2}$ units. The most intense peak attributable to the impacting particle is the feature at $91 \mathrm{amu}$, which is observed at low velocities $\left(v<\sim 7 \mathrm{~km} \mathrm{~s}^{-1}\right)$. This is diagnostic of the polystyrene core; it is well known that such aromatic compounds readily form the highly stable aromatic tropylium cation $\left(\mathrm{C}_{7} \mathrm{H}_{7}^{+}\right)$ species and produce a characteristic strong response at $91 \mathrm{amu}$ (Silverstein \& Bassler 1964). Indeed, in control experiments we have analysed relatively volatile styrene oligomers using conventional mass spectroscopy and also obtained strong signatures at 91 amu due to in-situ thermal degradation. This feature appears in both the PP3 spectra and also the slower PP4 events, although the many high mass features ( $>130 \mathrm{amu})$ are no longer present. There is a region at around $7.5-11 \mathrm{~km} \mathrm{~s}^{-1}$ where peaks become less frequent; at higher hypervelocities the 91 amu peak vanishes and does not reappear. This suggests that, at a threshold impact speed of approximately $8 \mathrm{~km} \mathrm{~s}^{-1}$, the kinetic energy of the impact is sufficient to destroy the tropylium cation species. A feature at $\sim 72$ amu gradually increases in intensity as the 91 amu species decreases. Once the impact speed exceeds $\sim 11 \mathrm{~km} \mathrm{~s}^{-1}$ the spectra fall into a new characteristic pattern, maintaining the standard separations of 12-14 amu between successive high mass peaks. Again, there is good agreement between different samples at comparable velocities. Once the impact velocity exceeds $\sim 17 \mathrm{~km} \mathrm{~s}^{-1}$ the periodic peaks disappear and the spectra become comparable to the relatively featureless spectra obtained for the PP sample.

The mass spectra can thus be sub-divided according to velocity (as indicated in the previous paragraph) into three distinct regimes: below $11 \mathrm{~km} \mathrm{~s}^{-1}, 11$ to $17 \mathrm{~km} \mathrm{~s}^{-1}$ and above $17 \mathrm{~km} \mathrm{~s}^{-1}$ (see Tables 5-7). The impact speeds used to delineate these regimes are approximate, and are probably accurate to within $1-2 \mathrm{~km} \mathrm{~s}^{-1}$. Occasional peaks observed outside the stated velocity regime are ignored; only typical behaviour is reported in Tables 5-7. Also, to a first approximation the contribution of the polypyrrole overlayer to the mass spectra is assumed to be negligible, since the latex projectiles comprise over $90 \%$ polystyrene by mass. Tentative assignments of the likely chemical species associated with each peak are then suggested. It should be remembered that the mass values referred to here are strictly mass/charge $(\mathrm{m} / \mathrm{z})$ ratios. It has been assumed that all species are singly charged ions $(z=1)$ when making the assignments. It is also noted that although mass 39 is traditionally considered as potassium (and is labelled as such here) it could also include a contribution from $\left[\mathrm{C}_{3} \mathrm{H}_{3}^{+}\right]$.

Even in the lowest velocity regime (Table 5), ionization is observed. The impact energy per projectile is sufficient to cause partial degradation of the polymer backbone, leading to chain scission of the relatively weak $\mathrm{C}-\mathrm{C}$ single bonds (for which the mean bond energy is around $350 \mathrm{~kJ}$ per mol of bonded carbon pairs). The high mass values that are observed are consistent with polyene fragments being formed of general formula $\left[\mathrm{CH}_{3}(\mathrm{CH}=\mathrm{CH})_{n}\left(\mathrm{CH}_{2}\right)_{m} \mathrm{CH}_{2}\right]^{+}$. For this homologous series the mass numbers $(\mathrm{MW})$ values are given by:

$M W=26 n+14 m+29$, where $n, m=0,1,2, \ldots$

This is illustrated in tabular form in Table 8. These assignments are consistent with the notion that the pendent phenyl rings of the polystyrene are mostly cleaved from the polymer 
Table 5. Mass spectra for impacts by polypyrrole coated polystyrene particles onto rhodium. Particle velocity $v<11 \mathrm{~km} \mathrm{~s}^{-1}$. * Note: there is no $\mathrm{Al}$ present in target or projectile and so peak at 27 has been attributed to a hydrocarbon species.

\begin{tabular}{|c|c|c|}
\hline$m / z$ & Ion & Tentative Structure \\
\hline 23 & $\mathrm{Na}^{+}$ & contaminant \\
\hline $27 *$ & {$\left[\mathrm{C}_{2} \mathrm{H}_{3}\right]^{+}$} & \\
\hline 39 & $\mathrm{~K}^{+}$ & contaminant \\
\hline 43 & {$\left[\mathrm{C}_{3} \mathrm{H}_{7}\right]^{+}$} & \\
\hline 51 & {$\left[\mathrm{C}_{4} \mathrm{H}_{3}\right]^{+}$} & {$[\mathrm{CH} \equiv \mathrm{CCH}=\mathrm{CH}]^{+}$} \\
\hline $56-57$ & {$\left[\mathrm{C}_{4} \mathrm{H}_{9}\right]^{+}$} & {$\left[\mathrm{CH}_{3} \mathrm{CH}=\mathrm{CHCH}_{2} \mathrm{CH}_{2}\right]^{+}$} \\
\hline $71-72$ & {$\left[\mathrm{C}_{5} \mathrm{H}_{11}\right]^{+}$} & {$\left[\mathrm{CH}_{3}\left(\mathrm{CH}_{2}\right)_{3} \mathrm{CH}_{2}\right]^{+}$} \\
\hline 91 & {$\left[\mathrm{C}_{7} \mathrm{H}_{7}\right]^{+}$} & tropylium ion \\
\hline \multirow[t]{2}{*}{103} & $\mathrm{Rh}^{+}$ & Target material \\
\hline & {$\left[\mathrm{C}_{8} \mathrm{H}_{7}\right]^{+}$} & {$\left[\mathrm{C}_{6} \mathrm{H}_{5} \mathrm{CH}=\mathrm{CH}\right]^{+}$} \\
\hline $115-116$ & {$\left[\mathrm{C}_{9} \mathrm{H}_{7}\right]^{+}$} & {$\left[\mathrm{C}_{6} \mathrm{H}_{5} \mathrm{C} \equiv \mathrm{CCH}_{2}\right]^{+}$} \\
\hline $127-129$ & {$\left[\mathrm{C}_{10} \mathrm{H}_{9}\right]^{+}$} & {$\left[\mathrm{C}_{6} \mathrm{H}_{5} \mathrm{C} \equiv \mathrm{CCH}_{2} \mathrm{CH}_{2}\right]^{+}$} \\
\hline $140-141$ & {$\left[\mathrm{C}_{11} \mathrm{H}_{9}\right]^{+}$} & {$\left[\mathrm{C}_{6} \mathrm{H}_{5} \mathrm{C} \equiv \mathrm{CCH}=\mathrm{CHCH}_{2}\right]^{+}$} \\
\hline $152-153$ & {$\left[\mathrm{C}_{11} \mathrm{H}_{21}\right]^{+}$} & {$\left[\mathrm{CH}_{2}=\mathrm{CH}\left(\mathrm{CH}_{2}\right)_{8} \mathrm{CH}_{2}\right]^{+}$} \\
\hline $166-167$ & {$\left[\mathrm{C}_{12} \mathrm{H}_{23}\right]^{+}$} & {$\left[\mathrm{CH}_{3} \mathrm{CH}=\mathrm{CH}\left(\mathrm{CH}_{2}\right)_{8} \mathrm{CH}_{2}\right]^{+}$} \\
\hline $178-179$ & {$\left[\mathrm{C}_{13} \mathrm{H}_{23}\right]^{+}$} & {$\left[\mathrm{CH}_{2}=\mathrm{CHCH}=\mathrm{CH}\left(\mathrm{CH}_{2}\right)_{8} \mathrm{CH}_{2}\right]^{+}$} \\
\hline 190-191 & {$\left[\mathrm{C}_{14} \mathrm{H}_{23}\right]^{+}$} & {$\left[\mathrm{CH}_{3}(\mathrm{CH}=\mathrm{CH})_{3}\left(\mathrm{CH}_{2}\right)_{6} \mathrm{CH}_{2}\right]^{+}$} \\
\hline 204 & {$[?]^{+}$} & ? \\
\hline (205) & {$\left[\mathrm{C}_{15} \mathrm{H}_{25}\right]^{+}$} & {$\left[\mathrm{CH}_{3}(\mathrm{CH}=\mathrm{CH})_{3}\left(\mathrm{CH}_{2}\right)_{7} \mathrm{CH}_{2}\right]^{+}$} \\
\hline 217 & {$\left[\mathrm{C}_{16} \mathrm{H}_{25}\right]^{+}$} & {$\left[\mathrm{CH}_{3}(\mathrm{CH}=\mathrm{CH})_{4}\left(\mathrm{CH}_{2}\right)_{6} \mathrm{CH}_{2}\right]^{+}$} \\
\hline 229 & {$\left[\mathrm{C}_{17} \mathrm{H}_{25}\right]^{+}$} & {$\left[\mathrm{CH}_{3}(\mathrm{CH}=\mathrm{CH})_{5}\left(\mathrm{CH}_{2}\right)_{5} \mathrm{CH}_{2}\right]^{+}$} \\
\hline 241 & {$\left[\mathrm{C}_{18} \mathrm{H}_{25}\right]^{+}$} & {$\left[\mathrm{CH}_{3}(\mathrm{CH}=\mathrm{CH})_{6}\left(\mathrm{CH}_{2}\right)_{4} \mathrm{CH}_{2}\right]^{+}$} \\
\hline \multirow[t]{2}{*}{$252-253$} & {$[?]^{+}$} & ? \\
\hline & {$\left[\mathrm{C}_{19} \mathrm{H}_{25}\right]^{+}$} & {$\left[\mathrm{CH}_{3}(\mathrm{CH}=\mathrm{CH})_{7}\left(\mathrm{CH}_{2}\right)_{3} \mathrm{CH}_{2}\right]^{+}$} \\
\hline $279-280$ & {$\left[\mathrm{C}_{21} \mathrm{H}_{27}\right]^{+}$} & {$\left[\mathrm{CH}_{3}(\mathrm{CH}=\mathrm{CH})_{8}\left(\mathrm{CH}_{2}\right)_{3} \mathrm{CH}_{2}\right]^{+}$} \\
\hline 312 & {$[?]^{+}$} & $?$ \\
\hline (315) & {$\left[\mathrm{C}_{24} \mathrm{H}_{27}\right]^{+}$} & {$\left[\mathrm{CH}_{3}(\mathrm{CH}=\mathrm{CH})_{11} \mathrm{CH}_{2}\right]^{+}$} \\
\hline
\end{tabular}

backbone, leaving fragments of the backbone that are highly unsaturated. It is also possible that some of these highly unsaturated fragments may be formed from the destruction of the aromatic rings. The most prominent feature is the mass peak at $91 \mathrm{amu}$, which corresponds to the well known tropylium cation. This aromatic seven-membered ring is particularly stable and is characteristic of the polystyrene component of the projectile. For some mass numbers it would be possible to make the assignment of a carbon cluster. However, whilst Kissel \& Krueger (2001), observe carbon clusters in negative ion mass spectra using similar particles, they comment that the production of such clusters is favoured for negative ions and thus such signals are not expected in positive ion mass spectra as obtained here.

In the intermediate velocity regime (Table 6), the impact energy per projectile is sufficient to destroy most of the larger molecular ions (i.e. most of the $\mathrm{C}-\mathrm{C}$ and $\mathrm{C}-\mathrm{H}$ bonds, and some of the stronger $\mathrm{C}=\mathrm{C}$ bonds, are now cleaved) and there is a general shift to lower mass peaks. It is not clear how to explain all the observed peaks. The peak that appears at mass 60-61 amu is one such case; a tentative suggestion is that it corresponds to a cluster of carbon atoms.

Finally, above $17 \mathrm{~km} \mathrm{~s}^{-1}$ (Table 7) we estimate that, even if most of the kinetic energy associated with the impinging projectile is dissipated through volatilization of the metal target,
Table 6. Mass spectra for impacts by polypyrrole coated polystyrene particles onto rhodium. Particle velocity $v=11-17 \mathrm{~km} \mathrm{~s}^{-1}$. * Note: there is no $\mathrm{Al}$ present in target or projectile and so peak at 27 has been attributed to a hydrocarbon species.

\begin{tabular}{|c|c|c|}
\hline$m / z$ & Ion & Tentative Structure \\
\hline 23 & $\mathrm{Na}^{+}$ & contaminant \\
\hline $27 *$ & {$\left[\mathrm{C}_{2} \mathrm{H}_{3}\right]^{+}$} & \\
\hline 39 & $\mathrm{~K}^{+}$ & contaminant \\
\hline 53 & {$\left[\mathrm{C}_{4} \mathrm{H}_{5}\right]^{+}$} & {$\left[\mathrm{CH} \equiv \mathrm{CCH}_{2} \mathrm{CH}_{2}\right]^{+}$} \\
\hline 55 & {$\left[\mathrm{C}_{4} \mathrm{H}_{7}\right]^{+}$} & {$\left[\mathrm{CH}_{3} \mathrm{CH}=\mathrm{CHCH}_{2}\right]^{+}$} \\
\hline $60-61$ & {$[?]^{+}$} & $?$ \\
\hline (71) & {$\left[\mathrm{C}_{5} \mathrm{H}_{11}\right]^{+}$} & {$\left[\mathrm{CH}_{3}\left(\mathrm{CH}_{2}\right)_{3} \mathrm{CH}_{2}\right]^{+}$} \\
\hline 72 & {$[?]^{+}$} & $?$ \\
\hline $82-83$ & {$\left[\mathrm{C}_{6} \mathrm{H}_{11}\right]^{+}$} & {$\left[\mathrm{CH}_{2}=\mathrm{CH}\left(\mathrm{CH}_{2}\right)_{3} \mathrm{CH}_{2}\right]$} \\
\hline 96-98 & {$\left[\mathrm{C}_{7} \mathrm{H}_{13}\right]^{+}$} & {$\left[\mathrm{CH}_{2}=\mathrm{CH}\left(\mathrm{CH}_{2}\right)_{4} \mathrm{CH}_{2}\right]^{+}$} \\
\hline 103 & $\begin{array}{l}\mathrm{Rh}^{+} \\
{\left[\mathrm{C}_{8} \mathrm{H}_{7}\right]^{+}}\end{array}$ & $\begin{array}{l}\text { Target material } \\
{\left[\mathrm{C}_{6} \mathrm{H}_{5} \mathrm{CH}=\mathrm{CH}\right]^{+}}\end{array}$ \\
\hline 107 & {$\left[\mathrm{C}_{8} \mathrm{H}_{11}\right]^{+}$} & {$\left[\mathrm{CH}_{3}(\mathrm{CH}=\mathrm{CH})_{3} \mathrm{CH}_{2}\right]^{+}$} \\
\hline $111-112$ & {$\left[\mathrm{C}_{8} \mathrm{H}_{15}\right]^{+}$} & {$\left[\mathrm{CH}_{3}(\mathrm{CH}=\mathrm{CH})\left(\mathrm{CH}_{2}\right)_{4} \mathrm{CH}_{2}\right]^{+}$} \\
\hline 117 & {$\left[\mathrm{C}_{9} \mathrm{H}_{9}\right]^{+}$} & {$\left[\mathrm{CH}_{2}=\mathrm{CH}\left(\mathrm{C}_{6} \mathrm{H}_{5}\right) \mathrm{CH}\right]^{+}$} \\
\hline 129 & {$\left[\mathrm{C}_{10} \mathrm{H}_{9}\right]^{+}$} & {$\left[\mathrm{C}_{6} \mathrm{H}_{5} \mathrm{C} \equiv \mathrm{CCH}_{2} \mathrm{CH}_{2}\right]^{+}$} \\
\hline $140-141$ & {$\left[\mathrm{C}_{11} \mathrm{H}_{9}\right]^{+}$} & {$\left[\mathrm{C}_{6} \mathrm{H}_{5} \mathrm{C} \equiv \mathrm{CCH}=\mathrm{CHCH}_{2}\right]^{+}$} \\
\hline
\end{tabular}

Table 7. Mass spectra for impacts by polypyrrole coated polystyrene particles onto rhodium. Particle velocity $v>17 \mathrm{~km} \mathrm{~s}^{-1}$. Note: there is no $\mathrm{Al}$ present in target or projectile and so peak at 27 has been attributed to a hydrocarbon species.

\begin{tabular}{lll}
\hline \hline$m / z$ & Ion & Tentative Structure \\
\hline 1 & $\mathrm{H}^{+}$ & \\
12 & $\mathrm{C}^{+}$ & \\
23 & $\mathrm{Na}^{+}$ & contaminant \\
$27 *$ & {$\left[\mathrm{C}_{2} \mathrm{H}_{3}\right]^{+}$} & {$\left[\mathrm{CH}_{2}=\mathrm{CH}\right]^{+}$} \\
39 & $\mathrm{~K}^{+}$ & contaminant \\
53 & {$\left[\mathrm{C}_{4} \mathrm{H}_{5}\right]^{+}$} & {$\left[\mathrm{CH} \equiv \mathrm{CCH}_{2} \mathrm{CH}_{2}\right]^{+}$} \\
55 & {$\left[\mathrm{C}_{4} \mathrm{H}_{7}\right]^{+}$} & {$\left[\mathrm{CH}_{3} \mathrm{CH}=\mathrm{CHCH}_{2}\right]^{+}$} \\
103 & $\mathrm{Rh}^{+}$ & Target material \\
& {$\left[\mathrm{C}_{8} \mathrm{H}_{7}\right]^{+}$} & {$\left[\mathrm{C}_{6} \mathrm{H}_{5} \mathrm{CH}=\mathrm{CH}\right]^{+}$} \\
\hline
\end{tabular}

there is sufficient residual energy to ensure cleavage of virtually all chemical bonds. Thus essentially all the spectral features in this velocity regime are best interpreted as elemental cations.

This interpretation of the evolution of the mass spectra with projectile velocity is based on chemical intuition and appears to be both logical and reasonable. It can be compared with standard positive ion mass spectra of polystyrene such as that of Brenna et al. (1991) obtained with laser microprobe and ion cyclotron resonance mass spectrometry. The mass spectra obtained in that work are very similar to that here from the low speed PP3 sample. There are many peaks starting at $m / z=91$. The peaks come in clusters, with the clusters separated by a mean of 10, 12 or 14 Daltons, and each cluster contains a series of peaks each separated by 1 Dalton from its neighbour. There are some differences however. For example, in the work of Brenna et al. (1991), the $m / z=91$ peak is observed but is not dominant, instead a cluster at $m / z=191-193$ is. Beyond this however the agreement is striking. 
Table 8. Possible mass numbers (amu) predicted by Eq. (3).

\begin{tabular}{ll|llllll}
\hline \hline & & & & $m$ & & \\
& & 0 & 1 & 2 & 3 & 4 & 5 \\
\hline \multirow{4}{*}{$n$} & 0 & 29 & 43 & 57 & 71 & 85 & 99 \\
& 1 & 55 & 69 & 83 & 97 & 111 & 125 \\
& 2 & 81 & 95 & 109 & 123 & 137 & 151 \\
& 3 & 107 & 121 & 135 & 149 & 163 & 177 \\
& 4 & 133 & 147 & 161 & 175 & 189 & 203 \\
& 5 & 159 & 173 & 187 & 201 & 215 & 229 \\
\hline
\end{tabular}

Hinz et al. (1994) report laser induced mass analysis of single particles of organic materials including polystyrene. They show a mass spectrum for positive ions from a 1.0 micron polystyrene particle. The peaks in the spectra are at low $m / z$ values, less than 120 . They assign the difference to the results of Brenna et al. (1991) to be the the result of using a greater laser irradiance. This is equivalent to increasing the impact speed in our experiments and their spectra are indeed similar in appearance to those obtained from the intermediate speed PP4 sample. There are again some differences, we see no cluster of peaks at $m / z=48-51$ and the peak they report at $m / z=39$ cannot be separated in our work from that of the known contaminant (potassium).

There are also impact ionization data for both positive and negative ion mass spectra for polyethylene-dioxythiophene coated polystyrene particles (Kissel \& Krueger 2001). In their positive ion mass spectra, at low speeds $\left(6 \mathrm{~km} \mathrm{~s}^{-1}\right)$ the only line they specifically associate with the particle (as against contaminants)is that at $m / z=91$ (the tropylium cation), they see no higher mass series of peaks. As impact speed increases they find that the line at $m / z=91$ disappears and a broad lower mass signal appears. Given that their mass resolution can easily permit resolution of integer mass species they attribute this to production of tropylium ions which possess sufficient internal excitation to decompose during the acceleration stage of their instrument. This they feel will result in a broad peak from $\mathrm{m} / \mathrm{z}=$ 80 to 90 . Hinz et al. (1994) do see structure between $\mathrm{m} / \mathrm{z}=$ 80-90, but they resolve it into individual integer peaks, so this is not the same feature. We have considered this when making a tentative identification of the $m / z$ values in Tables 5-7. Since our mass spectra at low speed appear similar to those of Brenna et al. (1991), we adopt the approach of attempting to assign an identification associated with the observed $\mathrm{m} / \mathrm{z}$ values and correlate these with either structure in the original particle or for example carbon clusters. We agree with Kissel \& Krueger (2001) in that at impact speeds between 10 and $20 \mathrm{~km} \mathrm{~s}^{-1}$ this structure changes, but noting the results of Hinz et al. (1994) we again make tentative chemical identifications based on the observed $\mathrm{m} / \mathrm{z}$ values rather than suggest the observation of unstable ions which decomposed during acceleration. It should be noted however, that both interpretations explain the change in the mass spectra above $\mathrm{m} / \mathrm{z}=100$ as the impact speed increases from 10 to $20 \mathrm{~km} \mathrm{~s}^{-1}$. It would be interesting to repeat the current experiments with a high resolution impact ionization detector (such as that of Kissel \& Krueger 2001) to see if the results favour the discrete $m / z$ values found for polystyrene by Hinz et al. (1994) or the broad peak type structure reported by Kissel and Krueger. However, since the current paper addresses a CDA type detector this is left for the future.

It is worth noting that, while the abundance of the sodium and potassium contaminants in these events is comparable to those obtained with iron projectiles, the abundance of the $27 \mathrm{amu}$ species is atypical, particularly at high velocities. It is possible that the lower density projectiles are less able to ionise this species to the same extent as iron. Similarly, it is not clear where rhodium appears within these spectra. At low velocities the response at $\sim 103$ amu in shape and amplitude resembles those surrounding it and happens to lie at the location of a predicted molecular species (i.e. the tropylium cation (91 amu) plus one carbon atom (12 amu). Once the impact velocity increases above $\sim 20 \mathrm{~km} \mathrm{~s}^{-1}$ a suitable candidate for rhodium appears; for iron projectiles this signal regularly appears above $12 \mathrm{~km} \mathrm{~s}^{-1}$. Krueger (1996) suggests that the relative abundance of projectile and target species within the mass spectrum can depend on the particle density. Our observation appears to support this hypothesis. Essentially, this suggests that the impact velocity thresholds for the production of various species may depend on the properties of the impactor.

In summary, these spectra provide interesting information concerning the formation and observation of impact-induced ionic plasmas. There are variations in spectral response at the same velocity but only in terms of the relative abundance of individual species. In general, the spectra obtained at a given velocity are self-consistent and reproducible over many impact events, although sometimes one or more individual species may disappear, probably due to slightly differing impact conditions.

It is possible to assign spectral features in the polypyrrolecoated polystyrene series of samples to the expected fragmentation chemistry of the organic projectiles. Furthermore, the evolution of the mass spectra with impact speed is clearly evident. The strong velocity dependence has important implications for the interpretation of data obtained from CDA-type instruments. It highlights the importance of knowing the impact speed for an individual event to enable a realistic, accurate interpretation of the mass spectrum.

\subsection{Identifying particle composition from the observed mass spectra}

The purpose of the Cassini CDA detector is to study the compositions of dust particles detected in space. There is no prior knowledge of the composition of any individual particle 
detected in space. It is therefore important to consider whether it is possible to classify the impacting particles into broad categories such as metal, organic or mineral, based solely upon the observed mass spectrum.

Firstly, we note that for organic materials the signals found via impact ionization and time-of-flight studies correspond to those expected from mass spectroscopy based upon other (i.e. thermal) decomposition methods. However, there is a marked dependence on impact speed. Effectively, as the speed (and hence specific impact energy) increases, the molecular bonds in the organic material are more readily broken. At lower speeds, many carbon bonds survive the impact, resulting in the formation of cationic organic fragments of relatively high mass. These fragments give rise to the periodic peaks in Fig. 10 (PP3) observed above the feature at $91 \mathrm{amu}$, which is attributed to the tropylium cation species. Each peak is separated by 12 to $14 \mathrm{amu}$ and the peaks are in repeating multiplets of four. While there is no reason to expect polystyrene latex particles to be found in space, if similar periodic patterns of higher mass fragments were observed for an impact event, then the inference of an organic-based micro-meteorite would be a reasonable interpretation. In particular, if a peak at 91 amu was obtained, an aromatic organic composition could be inferred with some confidence.

In contrast, iron microparticles produce mass spectra that, apart from the contaminants seen here, are relatively featureless. There are few peaks and no regular spacing between them. At first sight, this would seem to immediately allow clear differentiation from the organic projectiles. However, caution is required at high impact speeds (above $20 \mathrm{~km} \mathrm{~s}^{-1}$ ), since the mass spectra obtained from impact events using the PP5 and PP samples also become relatively featureless. This is not universally true for all materials. Impacts on the time-of-flight detectors on spacecraft that flew past Halley's comet in 1986 produced some spectra of great complexity, with higher mass signals of several hundred amu being observed. This encounter was at very high speed, so not all materials give just elemental signals in mass spectra derived from high energy impacts.

In the present study it has proved difficult to distinguish clearly between the mineral-based and iron projectiles simply from their observed mass spectra. The detailed discussion in the previous section was only possible because of the precise knowledge of the chemical composition of the microparticles. Without this information it would have been difficult to distinguish the mass spectra from AlSi events from those produced by impacts of iron particles.

\subsection{Mass resolution}

The largest polystyrene-based microparticles coated in polypyrrole (the PP3 sample) yielded spectra with many peaks, with features in excess of $250 \mathrm{amu}$. This enabled the first time an assessment to be made of the resolution of a CDA type instrument in the high atomic mass range. Figure 14 shows the mass resolution obtained from the individual peaks in the mean spectra of the iron and PP3 samples. The resolution of each peak is defined as $m / \Delta m$, where $m$ is the mean mass of a peak

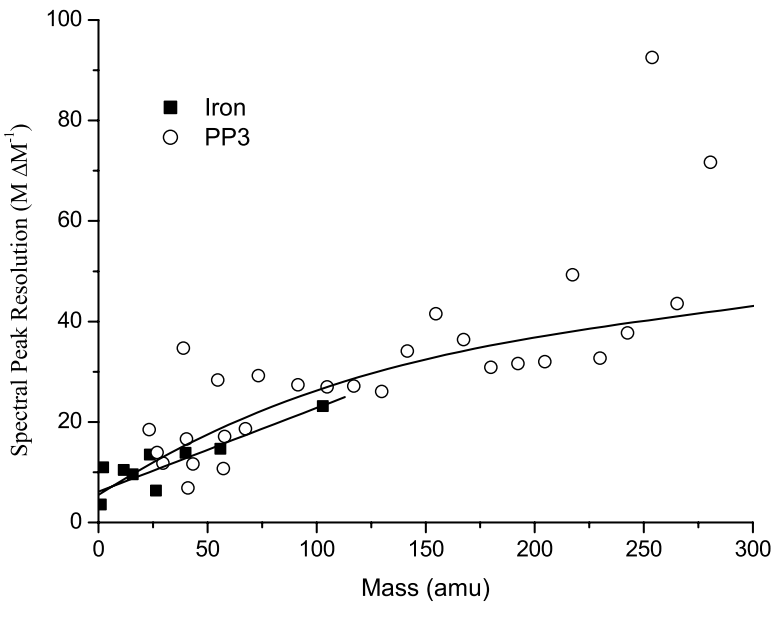

Fig. 14. Time-of-flight mass resolution for the iron and organic (PP3) microparticles.

and $\Delta m$ is given by the full-width-half-height of a Gaussian fit to the peak. The mass resolution data for the iron sample in Fig. 14 were fitted by a linear function that gave $m / \Delta m=$ $(6.1 \pm 1.5)+(0.17 \pm 0.03) m$. The PP3 data at similar masses were clearly compatible with this fit, as expected. The mass resolution for the PP3 data generally levels off at higher mass, although some high mass species exhibited very good resolution $(m / \Delta m>70)$. The resolution of the stable molecular species is surprisingly good considering the high probability of peak broadening due to additional or lost hydrogen atoms from the large molecules. Occasionally (e.g. at masses of approximately 38, 250 and $280 \mathrm{amu}$ ) some peaks have twice the mass resolution of their neighbours. The most likely explanation is that neighbouring signals have very similar masses that merge together to form the observed broad peak. Ignoring these occasional values, a fit to the PP3 data in Fig. 14 is shown (solid line) and yields $m / \Delta m=(5.5 \pm 6.6)+(0.3 \pm 0.2) m-$ $(8.10 \pm 0.01) \times 10^{-4} \mathrm{~m}^{2}$. This gives, for example, $\mathrm{m} / \Delta m=5$ for $\mathrm{H}^{+}$and 40 for $200 \mathrm{amu}$. However, it should be remembered that the higher mass peaks may represent signals from several neighbouring ion species that differ by a single $\mathrm{H}$ atom. The mass resolution is insufficient to resolve this. Therefore the true resolution is probably best represented by the occasional narrow peak, such as that at approximately 250 amu where the value of $m / \Delta m$ is much greater (of the order of 80-100).

It should also be noted that the time-of-flight data obtained here were recorded in the laboratory with standard electronics and no signal processing. Any space-deployed instrument will have its own custom-built electronics and signal processing which may change the mass resolution. The results obtained in this study should thus be treated as an indication of how the resolution evolves at high masses rather than absolute measures of the resolution for space instruments. The absolute resolution of the CDA flight instrument and electronics itself has previously been quoted as $m / \Delta m=20$ for $\mathrm{H}^{+}$and 50 for $\mathrm{Rh}^{+}$(Srama et al. 1996), i.e. approximately two to four times higher than that observed here. 


\section{Conclusions}

Several new types of microparticles that rely on the organic conducting polymer, polypyrrole, for their charging properties have been successfully accelerated using a 2 MV van de Graaff accelerator. This study has extended the particle compositions available for the laboratory calibration of dust analyser instruments based on impact ionization. The response to charging and acceleration of four low-density organic-based microparticles and aluminosilicate clay-based microparticles has been investigated in some detail and mass-velocity distributions were produced in the same mass-velocity regime as for that obtained with the more traditionally accelerated iron dust.

Within experimental scatter, the amplitude of the negative charge (normalised to particle mass) generated from impact on the rhodium target by all the new samples of microparticles concurs with that obtained from iron projectiles. This indicates that the difference in projectile density and composition (metallic, organic or mineral) has not significantly influenced the impact phenomena producing the observed ion total abundance. There are overall changes in the ionization yield behaviour with impact speed. These changes may be due to energy going into breaking of molecular bonds/melting/vaporization etc. rather than into ionization. There is some slight evidence that the transition speed between different different ionization regimes may be dependent on the projectile material. There is a clearer influence of projectile type on the rise time of the signals that are normally used to calculate the impact speed of the impinging projectile.

Complex, yet reproducible, time-of-flight spectra have been obtained for impacts of the conducting polymer-based microparticles on a rhodium target. In particular, the behaviour of three types of microparticles (two organic-based (PPY and PPY-PS) and one mineral-based (aluminosilicate clay)) has been studied and compared to that of iron. Many molecular fragments are displayed within the spectra derived from the organic-based projectiles, particularly at lower velocities $\left(v<15 \mathrm{~km} \mathrm{~s}^{-1}\right)$. The spectral response is consistent at specific velocities but the amplitudes of individual species can vary significantly, sometimes disappearing completely. Several elements (such as hydrogen, carbon, oxygen, and nitrogen) were only observed once a velocity threshold had been exceeded. Additionally, the time-of-flight spectra suggest that the aluminosilicate clay contains lithium. However, this feature was only observed within a certain velocity range $\left(7-16 \mathrm{~km} \mathrm{~s}^{-1}\right)$; the reason for this is unclear at present.

The spectra obtained from three polystyrene latexes of different particle sizes each coated with thin polypyrrole overlayers revealed an unexpectedly strong velocity dependence. It is emphasised that these spectra are consistent across different samples at comparable velocities and it is suggested that the spectra are dominated by features characteristic of the core latex, rather than the polypyrrole overlayer. However, the response changes significantly with increasing velocity. This highlights the importance of the accurate determination of impact velocity for individual events before accurate interpretation of the spectrum can be performed, particularly at low encounter velocities. Many higher mass molecular fragments have mass differences of 12 to 14 amu, suggesting the incremental addition of either $\mathrm{CH}$ or $\mathrm{CH}_{2}$ groups. It is tentatively suggested that, under certain impact conditions, this regularity might be used to identify an organic component for some micro-meteorites. Further work is planned that will use aliphatic polymer latex cores (rather than the aromatic polystyrene latex cores used in this study) to enable this hypothesis to be more fully tested. However, without a detailed prior knowledge of the chemical composition of the impinging particle and impact speed it was not possible to clearly distinguish between the mass spectra derived from the mineral particles used in this work and those obtained for iron projectiles. This was because both produced spectra dominated by relatively few peaks with low mass numbers.

The mass resolution of the CDA detector design was measured for high mass species ( $m>100 \mathrm{amu}$ ) in the low velocity time-of-flight spectra and shown to be non-linear at higher mass. However, this may be due to the production of cationic species in an event whose mass separation is less than the resolution of the detector at high masses.

Overall, the use of conducting polymers has greatly increased the range of particle compositions available for electrostatic acceleration. The new organic and organic-mineral projectiles are ideal for investigating plasma production on impact due to their ease of acceleration, highly variable chemical compositions and relatively narrow size distributions.

Acknowledgements. The work at the University of Kent was supported by a grant from the Particle Physics and Astronomy Research Council (UK) who also provided a Ph.D. studentship for B. J. Goldsworthy. We thank D. B. Cairns and S. A. Wilson for making some of the microparticles used. The synthesis of the conducting polymer-based microparticles at the University of Sussex was supported by the EPSRC, DSM Research (Geleen, The Netherlands) and the Defence and Evaluation Research Agency (Fort Halstead, UK).

\section{Appendix: Synthesis of polypyrrole-coated aluminosilicate particles}

The synthesis of the polypyrrole-coated aluminosilicate particles has not been reported previously and was carried out as follows. The raw China clay particles were supplied by English China Clay Ltd. The aluminosilicate particles were supplied suspended in an aqueous medium. The characteristics of the clay were determined in the laboratory. The density was $2660 \mathrm{~kg} \mathrm{~m}^{-3}$ and the conductivity was negligible $\left(<10-10 \mathrm{~S} \mathrm{~cm}^{-1}\right)$. SEM analysis showed a platelet morphology, polydisperse in size. A CHN elemental analysis gave C: $0.15 \%, \mathrm{H}: 1.45 \%, \mathrm{~N}: 0 \%$. The China clay particles were then suspended in de-ionised water. An oxidant was then added and, when fully dissolved, pyrrole was added. The resultant black dispersion was stirred for 24 hours and then centrifuged (at 4000 r.p.m. for $40 \mathrm{~min}$ ). The supernatant was replaced with de-ionised water and the centrifugation/re-dispersion repeated three times. The isolated black sediment was heated in an oven for 72 hours at $50{ }^{\circ} \mathrm{C}$ and then ground with a mortar and pestle to produce the dried, purified microparticles. The polypyrrole loading of the coated particles was determined by comparing their microanalytical nitrogen contents with those obtained 
for pure polypyrrole $(N=15.5 \% \mathrm{w} / \mathrm{w})$, given that the nitrogen content for the original un-coated clay particles was essentially zero.

\section{References}

Abel, M. L., Leadley, S. R., Brown, A. M., et al. 1994, Synth. Met., 66,85

Ahrens, T. J., Gupta, S. C., \& Jyoti, G. 2003. J. Geophys. Res., 108(E2), 5007, DOI: 10.1029/2002JE001912

Armes, S. P., \& Vincent, B. 1987, J. Chem. Soc., Chem. Commun., 288

Armes, S. P., Miller, J. F., \& Vincent, B. 1987, J. Colloid Inter. Sci., $118(2), 410$

Armes, S. P., Aldissi, M., Idzorek, G. C., et al. 1991, J. Colloid Inter. Sci., 141,119

Auer, S. 2001, in Interplanetary Dust, ed. E. Grün, B. A. S. Gustafson, \& H. Fechtig, 385

Bradley, J. P., Brownlee, D. E., \& Veblen, D. R. 1983, Nature, 301, 473

Brenna, J. T., \& Creasey, W. R. 1991. Appl. Spectroscopy, 45, 80

Burchell, M. J., Cole, M. J., \& McDonnell, J. A. M. 1998, Nucl. Instr. Meths. B, 143, 311

Burchell, M. J., Cole, M. J., McDonnell, J. A. M., et al. 1999a, Meas. Sci. Technol., 10, 41

Burchell, M. J., Cole, M. J., Lascelles, S. F., et al. 1999b, J. Phys. D: Appl. Phys., 32, 1719

Burchell, M. J., Willis, M. J., Armes, S. P., et al. 2002, Planet. Space Sci., 50, 1025

Dietzel, H., Eichhorn, G., Fechtig, H., et al. 1973, J. Phys. (E) Scientific Instrum., 6, 209

Divine, N. 1993, J Geophys. Res., 98, 17029

Friichtenicht, J. F. 1962, Rev. Sci. Inst., 33, 209

Friichtenicht, J. F., \& Slattery, J. C. 1963, NASA Technical Note D-2091

Goldsworthy, B. J., Burchell, M. J., Cole, M. J., et al. 2002, Adv. Space Res., 29, 1139

Grün, E., \& Staubach, P. 1996, in Astronomical Society of the Pacific Conf. Ser. 104, Physics, Chemistry, and Dynamics of Interplanetary Dust, ed. B. A. S. Gustafson, \& M. S. Hanner, 3

Grün, E., Fechtig, H., Hanner, M. S., et al. 1992a, Space Sci. Rev., 60, 317
Grün, E., Fechtig, H., Giese, R. H., et al. 1992b, A\&AS, 92, 411

Grün, E., Zook, H. A., Baguhl, M., et al. 1993, Nature, 362, 428

Grün, E., Krüger, H., Graps, A. L., et al. 1998, J. Geophys. Res., 109, 20011

Grün, E., Landgraf, M., Horanyi, M., et al. 2000, J. Geophys. Res., 105,10403

Hansen, D. O. 1968, Appl. Phys. Lett., 13, 89

Hinz, K.-P., Kaufman, R., \& Spengler B. 1994, Anal. Chem., 66, 2071

Keaton, P. W., Idzorek, G. C., Rowton, L. J., et al. 1990, Int. J. Impact Engng., 10, 295

Khan, M. A. 2001, Synthesis, characterisation and applications of conducting polymer-coated latexes, D.Phil. Thesis, University of Sussex, UK

Khan, M. A., \& Armes, S. P. 2000, Adv. Mater., 12, 671

Kissel, J. 1986, ESA SP-1077, 67

Kissel, J., \& Krueger, F. R. 1987, Appl. Phys. A, 42, 69

Kissel, J., \& Krueger, F. R. 2001, Rapid Communications in Mass Spectrometry, 15, 1713

Kissel, J., Sagdeev, R. Z., Bertaux, J. L., et al. 1986a, Nature, 321, 280

Kissel, J., Brownlee, D. E., Büchler, K., et al. 1986b, Nature, 321, 336

Knabe, W., \& Krueger, F. R. 1982, Naturforsch, 37a, 1335

Krueger, F. R. 1996, Adv. Space Res., 17(12), 1271

Lascelles, S. F., \& Armes, S. P. 1995, Adv. Mater., 7, 864

Lascelles, S. F., \& Armes, S. P. 1997, J. Mater. Chem., 7, 1339

Matney, M. J., \& Kessler, D. J. 1996, in Astronomical Society of the Pacific Conf. Ser. 104, Physics, Chemistry, and Dynamics of Interplanetary Dust, ed. B. A. S. Gustafson, \& M. S. Hanner, 15

Ratcliff, P. R., Burchell, M. J., Cole, M. J., et al. 1997, Int. J. Impact Eng., 20, 663

Silverstein, E. M., \& Bassler, G. C. 1964, Spectrometric Identification of Organic Compounds (John Wiley and Sons, Inc.)

Spehalski, R. J. 1996, Proc. Int. Soc. Optical Engineering (SPIE) 2803, ed. L. Spilker, 2

Srama, R., \& Grün, E. 1996, in Astronomical Society of the Pacific Conf. Ser. 104, Physics, Chemistry, and Dynamics of Interplanetary Dust, ed. B. A. S. Gustafson, \& M. S. Hanner, 227

Srama, R., Bradley, J. G., Grün, E., et al. 2003, Space Sci. Rev., in press

Stübig, M., Grün, E., \& Srama, R. 2003, Space Sci. Rev., in submission 